\title{
SUPERDEFORMATIONS AND FERMION DYNAMICAL SYMMETRIES
}

\section{Cheng-Li WU}

Department of Physics and Atmospheric Science, Drexel University, Philadelphia, PA 10104; Department of Physics and Astronomy, University of Tennessee, Knoxville, TN 37996; Joint Institute for Heavy-Ion Research, Oak Ridge National Laboratory, TN 37831

\begin{abstract}
In this talk, I will present a link between nuclear collective motions and their underlying fermion dynamical symmetries. In particular, I will focus on the microscopic understanding of deformations. It is shown that the $\mathrm{SU}_{3}$ of the one major shell fermion dynamical symmetry model (FDSM) is responsible for the physics of low and high spins in normal deformation. For the recently observed phenomena of superdeformation, the physics of the problem dictates a generalization to a supershell structure (SFDSM), which also has an $S U_{3}$ fermion dynamical symmetry. Many recently discoverea features of superdeformation are found to be inherent in such an $\mathrm{SU}_{3}$ symmetry. In both cases the dynamical Pauli effect plays a vital role. A particularly noteworthy discovery from this model is that the superdeformed ground band is not the usual unaligned band but the D-pair aligned (DPf.) band, which sharply crosses the excited bands. The existence of such DPA band is a key point to understand many properties of superdeformation. Our studies also pose new experimental challenge. This is particularly interesting since there are now plans to build new and exciting $\gamma$-ray detecting systems, like the GAMMASPHERE, which could provide answers to some of these challenges.
\end{abstract}

\section{INTRODUCTION}

I am indesd honored to be chosen by the organizing committee of this joyous conference to give an invited talk on the Fermion Dynamical Symmetry Model (FDSM) ${ }^{1}$. Since Professor Arima is one of the pioneers in the concept of nuclear dynamical symmetries, it is particularly appropriate that the talk is given as a tribute to his scientific career. I am also happy that Professor Twin has just given a beautiful overview of the current experimental situation for superdeformation. I would like to use this opporturity to discuss our recent theoretical understanding of four major questions (see section 3) in this field. The work on superdeformation is in collaboration with Da Hsuan Feng (Drexel University) and Mike W. Guidry (University of Tennessee).

The FDSM was proposed by Da Hsuan Feng, Mike W. Guidry, Jin-Quan Chen (Nanjing University), Xuan-Gen Chen (Drexel University) and myself. Also, Hua Wu, Wei-Min Zhang, Zhen-Ping Li, Xiao-Ling Han,Lian-Ao Wu, Xing-Wang Pan, Zhi-Min Lü, Guang-Zhou Liu, Jia-Lun Ping and Joe Ginocchio have all contributed significantly.

Our physical world is often understood by symmetry considerations. For example, space-time symmetry, which is the invariance of a system under space-time 
transformation, had led us to fundamental conservation laws like energy-momentum or angular momentum. More generally, symmetry may be defined as the invariance of a system's Hamiltonian under certain group operations. Our concern here will be with dynamical symmetry, which is the hierarchical invariance of a system. When a system possesses dynamical symmetry, its Hamiltonian is a function of the Casimir invariants of a particular group chain. In the past few decades, this important conce:pt has blos:omed and is now broadly utilized in many areas of physics (for a comprehensive survey, see ref. 2 ).

In nuclear physics, there is an obvious advantage in using dynamical symmetry, because if the complicated nuclear many-body Hamiltonian possesses a dynamical symmetry, then with the aid of group theory, one can solve the Schrodinger equation analytically. In practice, however, a realistic system rarely possesses perfect symmetry. Therefore, it is desirable to separate the full Hamiltonian into a dynamical symmetry part and a symmetry breaking part. The former could represent a particular nuclear collective mode (referred to as just "collectivity" from now on), while the latter represents deviations from "benchmark" symmetries. If such a separation is possible, then not only will the many-body problem be greatly simplified, but one may gain deeper insights by studying the aralytical solutions offered by group theoretical means.

The pioneering idea of dynamical symmetry in collectivity can be traced to Elliott's $\mathrm{SU}_{3}$ work in the late fifties, ${ }^{3}$ which establishes a profound connection in the spherical $s d$ shell model between the geometry of rotation and an underlying $S_{3}$ dynamical symmetry. While Elliott's model cannot be applied to other physical shells, an ingenious reformulation was proposed simultaneously a decade later (1969) by Arima, Harvey and Shimizu, 4 and Hecht and Adler 5 . This is the pseudo-SU $U_{3}$ model, which introduces a 1/2 pseudo-spin (not to be confused with the real spin), and is designed for deformed nuclei in regions other then the $s d$ shell. Later, Rosensteel and Rowe 6 proposed a $2 \hbar \omega$ excitation $S p(3, R)$ symplectic model, which has Elliott's $S U_{3}$ as its maximal compact subgroup. Recently, Draayer et al. furthered these developments to a pseudo symplectic model, thus allowing multi-shell excitations to take place in the pseudo-SU scheme.7

Another important development of nuclear dynamical symmetry which is particularly relevant to the subject matter of my talk is the Interacting Boson Model (IBM). 8 Again, Professor Arima is an author of this important model, which differs considerably in style and philosophy from the aforementioned models. Instead of a mono-chain $\left(\mathrm{SU}_{3}\right)$ symmetry, the IBM starts from a highest boson symmetry $U(\sigma)$ and explores a multi-chain dynamical symmetry. Each chain of the model is anchored in a physically realizable collective mode: $O_{6} \leftrightarrow \gamma$-soft; $U_{5} \leftrightarrow$ vibration; $S U_{3} \leftrightarrow$ rotation. It also establishes the importance of $S(J=0)$ and $D(J=2)$ identical nucleon pairs, simulated as $s$ and $d$ bosons, in allowing these dynamical symmetries to emerge. 
The next important milestone, in chronology and substance, for multi-chain nuclear dynamical symmetries is the work by one of the conference organizers, Joe Ginocchio. The Ginocchio Model resides in the fermion space and possesses many dynamical symmetries (1978). ${ }^{9}$ The coherent $S$ and $D$ pairs of the model and the corresponding multipole operators are constructed via the so-called $k-i$ basis, $b_{k m_{k} i m_{\mathrm{i}}}^{\dagger}$ with $i=3 / 2$ or $k=$ 1 . The precise definitions of these operators are as follows:

$$
\begin{aligned}
& b_{k m_{k} i m_{i}}^{\dagger}=\sum_{j}<k m_{k} i m_{i} / j m>a_{j m}^{\dagger} \\
& \left.S^{\dagger}=\sqrt{\Omega_{k i} i 2\left[b_{k i}^{\dagger}\right.} b_{k i}^{\dagger}\right]_{\infty 0}^{\infty 0} \quad \text { (for any } k \text { and } i \text { ) }
\end{aligned}
$$

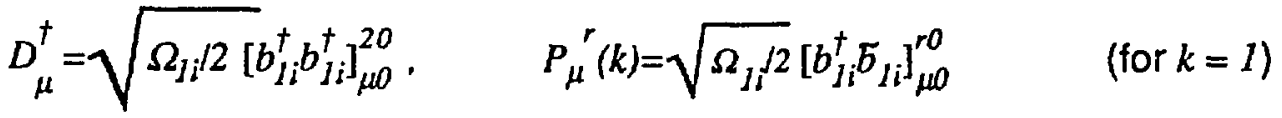

$$
\begin{aligned}
& \left.D_{\mu}^{\dagger}=\sqrt{\Omega_{k 3 / 2} / 2\left[b_{k 3 / 2}^{\dagger}\right.} b_{k 3 / 2}^{\dagger}\right]_{0 \mu}^{02}, P_{\mu}^{r}(i)=\sqrt{\Omega_{k 3 / 2} / 2}\left[b_{k 3 / 2}^{\dagger} \sigma_{k 3 / 2}\right]_{0 \mu}^{O r} \quad(\text { for } i=3 / 2)
\end{aligned}
$$

where $a_{j m}^{\dagger} 10>$ is a single-particle state and $\Omega_{k i}=(2 k+1)(2 i+1) / 2$ is the shell pair degeneracy. Remarkably, these operators are closed algebraically. The resulting dynamical groups are $\mathrm{Sp}_{6}$ (for $k=1$ ) or $\mathrm{SO}_{8}$ (for $i=3 / 2$ ). Indeed, if the nuclear two body Hamiltonian is a scalar in these groups, it can then be written as

$$
H=G_{0} S^{\dagger} S+G_{2} D^{t} D+\sum_{r} B_{r} P^{r} \cdot P^{r}
$$

Dynamical symmetries of such a system are depicted in Fig. 1. What is in my opinion profound is that all the IBM dynamical symmetries are found in the more fundamental fermionic level and each corresponds to a known collective mode (see Table 1).

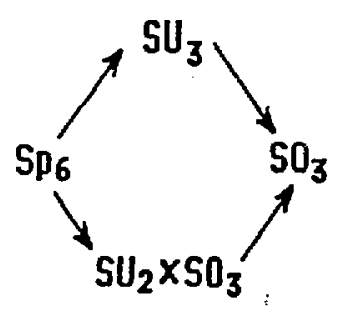

(a)

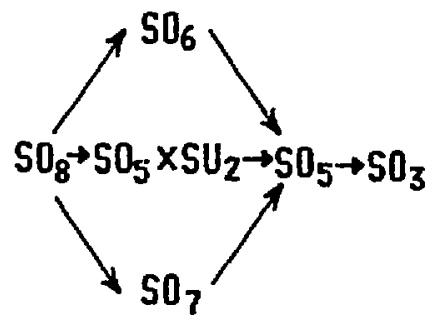

(b)

Fig. 1 The group chains of $\mathrm{Sp}_{6}$ and $\mathrm{SO}_{8}$

$$
\text { (a) } k=1 \text {; (b) } i=3 / 2
$$

Table 1. Fermion dynamical symmetries and the various collective modes

\begin{tabular}{|lllll|}
\hline \multicolumn{1}{|c}{ FDS } & Colloctive mode & & $1 B M$ \\
\hline$S P(6) \supset S U(3)$ & $\leftrightarrow$ & Roletion & $\leftrightarrow$ & $U(6) \supset S U(3)$ \\
$S O(8) \supset S O(6)$ & $\leftrightarrow$ & $Y$-SOIt & $\leftrightarrow$ & $U(6) \supset S O(6)$ \\
& & & & \\
$S P(6) \supset S U(2) \times S O(3)$ & & & & \\
$S O(8) \supset S O(5) \times S U(2)$ & $\leftrightarrow$ & Vibration $\leftrightarrow$ & $U(6) \supset U(5)$ \\
$S O(8) \supset S O(7)$ & & & & \\
\hline
\end{tabular}


Although the Ginocchio mode! is an intellectual tour-de-force, as Professor M. W. Kirson of the Weizmann Institute calls it, 10 there were some apparent difficulties to consider it a realistic nuclear model. The most glaring one (and most irtriguing in hindsight) is associated with the Pauli principle. It is manifested in the $S p_{6} \supset S U_{3}$ chain, where some $\mathrm{SU}_{3}$ irreducible representations (irreps) are Pauli forbidden. For example, the Pauli principle will forbid the occurrence of $S U_{3}(\lambda, \mu)$ irreps if $\lambda+\mu>2 \Omega_{k i} / 3$. Therefore, when the valence nucleon number $n$ exceeds $2 \Omega_{k i} / 3$, the symmetric irrep $(n, 0)$ (normally assigned as the ground band) is Pauli-forbidden. This implies that a straightforward application of the model to real nuclei will run afoul of the fact that the vast majority of well-deformed nuclei do occur near the mid-physical shell. This led Ginocchio to abandon the $S p_{6}$ model, and therefore its $S U_{3}$ limit. Other difficulties in the model are the opaque meaning of the $k-i$ decomposition, and the unclear relationship between the $k-i$ and the shell model basis. Therefore, the Ginocchio model does not appear to have the necessary ingredients to be a model for real nuclei.

In 1986, we proposed the Fermion Dynamical Symmetry Model (FDSM). ${ }^{1}$ As we shall see, it appears to be the next logical step in the establishment of a realistic and fermion-based nuclear model, and also taps the wisdom of the multi-chain concept. The FDSM can be regarded as a one-major spherical shell model. A salient feature is that the truncation for the normal-parity levels is carried out using the Ginocchio $S$ and $D$ space, while the $S$ pair condensate provides the truncation scheme for the abnormal level. Indeed, within a major physical shell, one can uniquely establish a unitary transformation between the $k-i$ and the shell model basis. This led us to reclassify the shell model single-particle basis. The reclassification and the corresponding symmetries for the $S-D$ subspace are shown in Table 2. In addition, because of the existence of the abnormal level, one finds that the $\mathrm{SU}_{3}$ limit of the Ginocchio model is resurrected. This can be understood by the following consideration. The FDSM SU $U_{3}$ symmetry comes from the $n_{1}$ normal-

\begin{tabular}{|c|c|c|c|c|c|c|c|c|}
\hline be. & 1 & 2 & 3 & 4 & 5 & 6 & 7 & 8 \\
\hline N & 0 & 1 & 2 & 3 & 34 & 45 & 556 & $\begin{array}{lll}6 & 6 & 7\end{array}$ \\
\hline$k$ & 0 & 1 & 1 & 0 & 10 & 20 & 110 & 110 \\
\hline i & $\frac{1}{2}$ & $\frac{1}{2}$ & $\frac{3}{2}$ & $\frac{7}{2}$ & $\frac{3}{2} \frac{9}{2}$ & $\frac{3}{2} \frac{11}{2}$ & $\frac{1}{2} \frac{7}{2} \frac{13}{2}$ & $\begin{array}{lll}\frac{3}{2} & \frac{9}{2} & \frac{15}{2} \\
\end{array}$ \\
\hline & & $A / 2$ & $\begin{array}{l}s_{1 / 2} \\
d_{3 / 2} \\
d_{s / 2}\end{array}$ & & $\begin{array}{l}D_{1 / 2} g_{9 / 2} \\
a_{3 / 2} \\
f_{5 / 2}\end{array}$ & $\begin{array}{l}5 / 2 \\
I / 2 \\
I\end{array}$ & & $\begin{array}{l}s_{1 / 2} g_{7 / 2} j_{k} \\
d_{3 / 2} j_{9 / 2} \\
d_{5 / 2} i_{i / 2}\end{array}$ \\
\hline 5 & & & $65_{5} f_{3}$ & & 6883 & $B_{8}$ & ${ }_{6}^{6}$ & 66 \\
\hline 0 & 0 & 0 & 0 & 0 & 5 & 6 & 7 & 8 \\
\hline & & 3 & 6 & 4 & 6 & 10 & 15 & 21 \\
\hline & 2 & 8 & 20 & 20 & 50 & 82 & 126 & 184 \\
\hline
\end{tabular}

No. labels the shell ordering; $N, k, i$ label the principle, pseudo-orbit and pseudo-spin quantum numbers; $\Omega_{0}$ and $\Omega_{1}$ are the pair degeneracies of the abnormal-parity and normal-parity levels for each shell. The number $n$ is the maximum allowable nucleon number up to and including tha! particular inajor shell. The symbols $G_{6}, G_{8}$ and $G_{3}$ are short-hand notation for the symmetries in the $S-D$ subspace $: G_{6}=\left(S p_{6} \times S U_{2}\right) G_{8}=\left(S O_{8} \times S U_{2}\right), G_{3}=\left(S U_{3} \times S O_{6}\right)$. 
parity particles. Therefore it is certainly possible that the total valence nucleon number $n$ $=n_{1}+n_{0}$, where $n_{0}$ is the particle number in the abnormal-parity level can approach midshell, while $n_{1}$ is still less than $2 \Omega_{1} / 3$. Once this was realized, it led immediately to the establishment of a microscopic (namely fermionic) nuclear modal with multichain dynamical symmetries. Very recently, stimulated by the exciting discovery of superdeiormation, this model was further extended to include multi-shell configurations. This extension has led us to the supershell FDSM (SFDSM). Although it still is in its infancy, the SFDSM already appears to encompass much of the physics of superdeformation.

In the following, I will first discuss how the one-major shell FDSM accounts for the physics of normal deformation in low-and high-spin phenomena. Then In section 3, I will discuss the SFDSM. Finally, in section $4 \mathrm{I}$ will illustrate how the basic features of superdeformed nuclei emerge from the supershell $\mathrm{SU}_{3}$ dynamical symmetry.

\section{THE ONE-MAJOR-SHELL FDSM AND NUCLEAR NORMAL DEFORMATIONS}

In the past few years the FDSM was examined extensively. It was demonstrated that the phenomenological IBM is a large shell limit $\left(\Omega_{1} \rightarrow \infty\right)$ of the FDSM, and the Geometrical Model (more precisely the Particle-Rotor Model (PRM)) was a large particle number limit $\left(n_{1} \rightarrow \infty\right) .11,12$ In addition, using the well-known coherent states approach, 13 the connection between the FDSM and the traditional HFB approach was established. ${ }^{14}$ The interrelationships among the models are shown schematically in Fig. 2, which suggests that the FDSM provides a unified shell model basis for both the boson and geometrical pictures. Of course, neither $\Omega_{1}$ nor $n_{l}$ are really infinite. Hence, the Pauli effect and the finite particle number effect must have significant consequences. Indeed, they have led to many new predictions and understandings of nuclear systematics. These include a new vibrational $\mathrm{SO}_{7}$

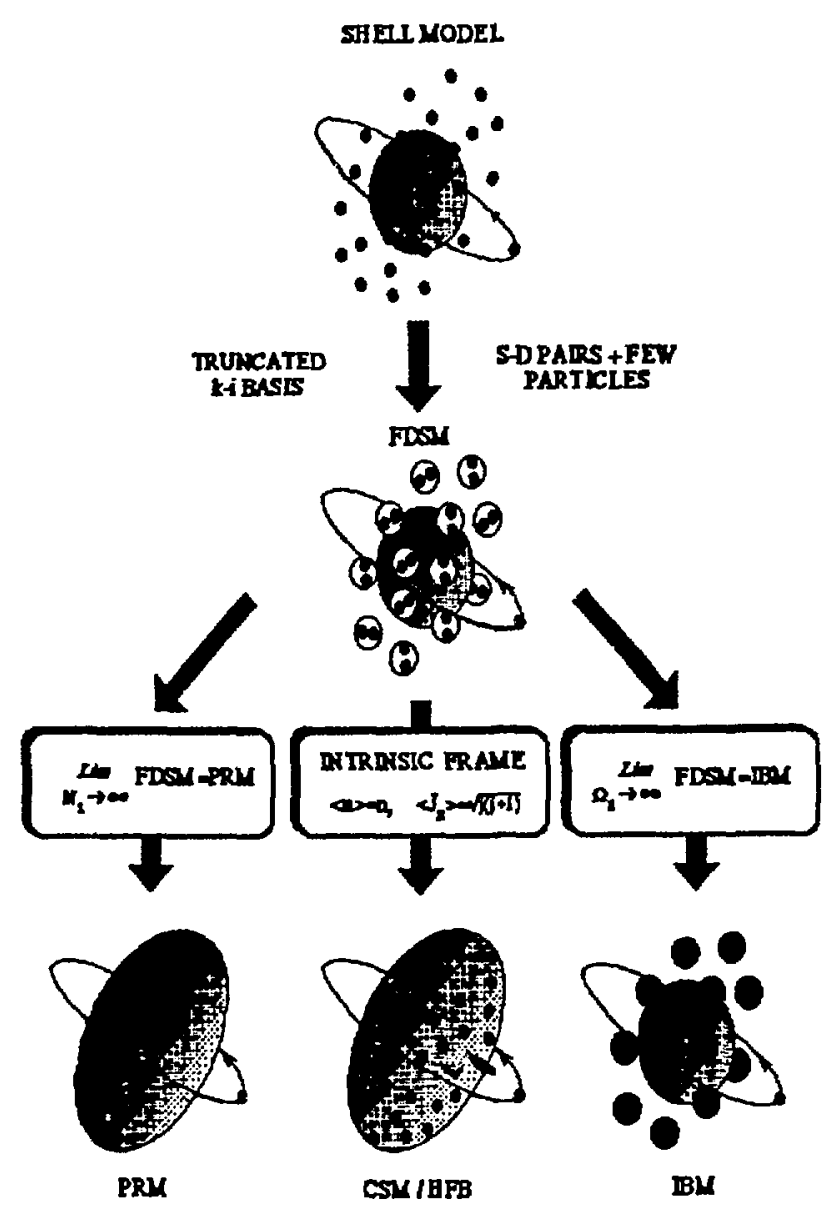

Fig. 2 The relationship between the FDSM and other models 
mode in the $R u$-Pd region, 15 the mid-shell saturation of the $B(E 2)$ 's in heavy nuclei, 16 actinide mass anomalies, 17 the loss of $B(E 2)$ strengths and the termination of bands in high-spin spectroscopy, 18 the necessity of Coriolis attenuation in the PRM, ${ }^{19}$ the suppression of two neutron transfer at low spins, 20 and so on.

Another important feature of the FDSM is that it can accommodate states in which nucleons do not form the coherent $S$ and $D$ pairs of Eqs. (1.2)-(1.4). The number of such unpaired nucleons is what we call the heritage quantum number $u$. By allowing $u$ $\neq 0$, the FDSM can account for not just even-even nuclei at low-spin, but oddnucleon system and high-spin physics as well. By including all possible $u$ 's, a prospect manageable only for sufficiently small spaces (e. g., sd shell nuclei or nuclei very close to doubly closed shells), the entire shell model space is recovered. However, for nuclei

(a) The PRM calculations

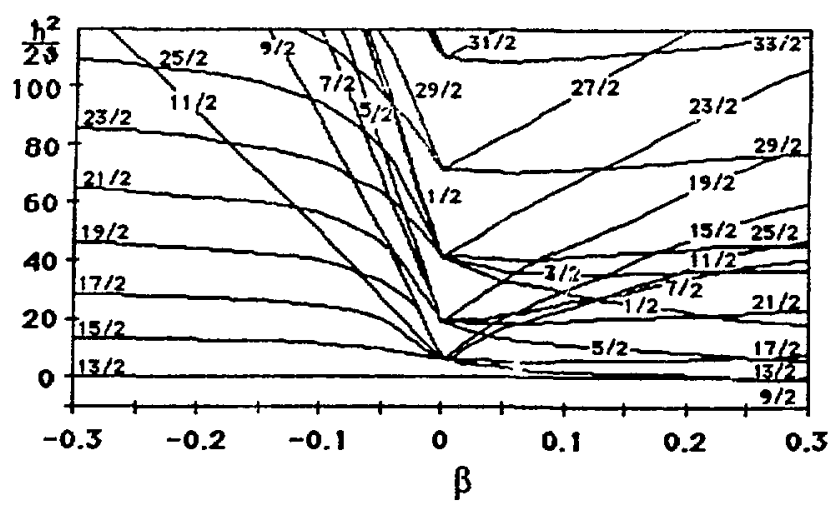

(b) The FDSM calculations

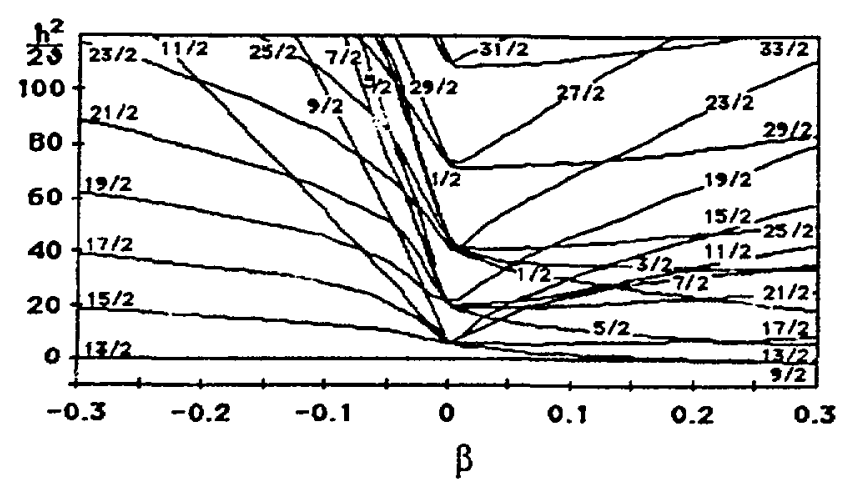

Fig.3 Model calculations of the $i_{13 / 2}$ band for an odd system.

The spectrum is plotted as $[E(J)-E(13 / 2)] /\left(\hbar^{2} / 2 g\right)$. In the FDSM plot $\chi$ is converted into $\beta$ via the relation $\beta=\chi_{n_{1}} / \sqrt{ } 2$. In both calculations, only one particle is assumed in the abnormal Parity $i_{13 / 2}$ shell. far from closed shells, where the ordinary shell model is impotent, the FDSM can be employed as a truncation scheme by limitinr $u$ to a tractable number, say $u \leq 3$, so that spherical shell model calculations for heavy nuclei become feasible. Thus, the FDSM provides a unified microscopic description of low energy nuclear structure from light to heavy systems, and from low to high-spins.

In this talk, I shall mainly discuss the connection between the FDSM and the geometrical description; in particular,I will emphasize the microscopic understanding of deformation from the fermion dynamical symmetry view point. Before discuss the deformation, let me try to convince you by a calculation that there indeed is a close relationship between the FDSM and the PRM. The results are displayed in Fig. 3. This is a comparison between a PRM calculations using the standard PRM code and FDSM calculations in which the $\mathrm{SU}_{3}$ Hamiltonian was diagonalized numerically with a quadrupole 
coupling term between the $S-D$ pairs and an odd particle in the abnormal-parity level. Both caiculations assumed the odd particle to reside in the $i_{13 / 2}$ orbital. For the FDSM, there are 16 nucleons (8 pairs) in the normal-parity levels $\left(n_{1}=16\right)$. (For details see ref. 12). As is obvious from Fig. 3 , there is extremely good agreement for these two calculations. It should be noted that in the strong coupling limit (around $\beta \approx-0.3$ ), one does find some small differences which has been shown to be finite particle effects. Hence, we can safely conclude that in the $n_{1} \rightarrow \infty$ limit, the PRM and the FDSM are equivalent.

Furthermore, I would like to emphasize that the FDSM may be regarded as a microscopic particle-rotor model. This can be seen as follows: the $S p_{6}$ FDSM Hamiltonian can formally be written as a PRM-like Hamiltonian:

$$
H=H_{s . p .}+H\left(n_{\gamma} n_{\beta}\right)+V_{p}+\frac{1}{2 g_{r i g}} L^{2}+\delta L I+\gamma^{2}+H_{o}\left(n_{l}, n_{0}\right)
$$

In Eq. (2.1), $H_{\text {s.p. }}$ is the deformed single-particle energy, $H\left(n_{\gamma}, n_{\beta}\right)$ the $\beta-\gamma$ vibrational energy, $V_{p}$ the pairing energy, $L^{2} / 2 g_{\text {rig }}$ the energy of the rotating "core", and $I$ the alignment angular momentum. Each term in the FDSM particle-rotor Hamiltonian is now defined microscopically: the "core" angular momentum $L$ is the angular momentum of the $S$ - $D$ pairs in the normal-parity levels, the alignment $I$ is the total psuedo-spin, and

$$
\begin{gathered}
\boldsymbol{H}_{s . p .}=\left\langle\sum_{j} e_{j} n_{j}-\chi m \omega_{0}^{2} r^{2} P^{2} \cdot q^{2}>_{\text {intrinsic frame }}=\sum_{K} \varepsilon_{K}(\beta) n_{K},\right. \\
H\left(n_{\gamma s} n_{\beta}\right)=-B_{2}^{\pi v} C(\lambda, \mu)=-B_{2}^{\pi v} C\left(n_{1}, 0\right)+h \omega\left(n_{\gamma}+n_{\beta}+K / 2\right)+O\left(1 / n_{l}\right), \\
h \omega=3 B_{2}^{\pi v} n_{1}, \quad n_{\gamma}=\left(n_{1}-\lambda-2 \mu\right) / 3, \quad n_{\beta}=(\mu-\kappa) / 2, \quad K=\kappa, \\
\frac{1}{2 g_{r i g}}=\frac{3}{8} B_{2}^{\pi v}, \quad C(\lambda, \mu)=\left(\lambda^{2}+\mu^{2}+\lambda \mu+3 \lambda+3 \mu\right) / 2
\end{gathered}
$$

In Eq. (2.2) the $e_{j}$ 's are the spherical single-particle energies, $\chi \boldsymbol{P}^{2}\left(P^{2}=P^{2 \pi}(k)+P^{2 v}(k)\right)$ corresponds to the collective quadrupole operator $\alpha_{2 \mu}$ in the geometrical model, and $q^{2}$ is the noncollective single-particle quadrupole operator, which is defined as $Y_{2 \mu}$ with the collective component removed (i.e. the part with $i$ coupled to zero ). In Eq. (2.3), $C(\lambda, \mu)$ is the expectation value of the $S U_{3}$ Casimir operator and $B_{2}^{\pi v}$ is the $n-p$ quadrupolequadrupole interaction strength. It has been shown that there exists a one-to-one correspondence between different $S U_{3}$ irreps, characterized by $(\lambda, \mu) \kappa$, and the $\beta-\gamma$ vibrational states, which are characterized by the quantum numbers $\left(n_{\gamma}, n_{\beta}\right) K .21$ The terms $H_{0}\left(n_{1}, n_{0}\right), \delta L \cdot I$, and $\gamma^{2}$ are not present in the phenomenological PRM. $H_{0}\left(n_{1}, n_{0}\right)$ comes from the monopole-monopole interactions (i. e. the $r=0$ components in Eq.(1.5)), and is a function of $n_{1}$ and $n_{0}$; it affects only the band head energies. The terms $\delta L \cdot I$ 
and $\gamma^{2}$ originate from the spin-spin and spin-orbit interactions (i. e. the $r=1$ components in Eq.(1.5)); they will modify the Coriolis interaction and the recoil term. These terms can be regarded as a "microscopic" modification of the phenomenological PRM.

Ti:re are also other very interesting physical consequences. For example, strictly spealing, the pairing interaction is an $\mathrm{SU}_{3}$ symmetry breaking term. However, for nuclei far from closed shells the quadrupole-quadrupole interaction will dominate, and thus $\mathrm{SU}_{3}$ appears to be a good symmetry. Hence the off-diagonal pairing matrix elements will play a minor role and may be neglected as an approximation. With only the diagonal pairing matrix element included, we notice that:

$$
\begin{gathered}
<V_{p}>=V_{p}(L=0)+F\left(n_{1}\right) L(L+1), \\
F\left(n_{1}\right)=\sum_{\sigma=\pi ; v}\left|G_{0}^{\sigma}-G_{2}^{\sigma} \frac{2 \Omega_{1} \sigma_{i 3-n_{l}} \sigma_{+2}}{4 n_{l}\left(n_{l}-1\right)} n_{1}{ }^{\sigma}=\right| \overline{G_{0}-G_{2}} \mid \frac{1}{2\left(n_{l}-I\right)} \quad\left(\text { for }\left(n_{1}, 0\right)\right. \text { irrep) }
\end{gathered}
$$

and therefore Eq. (2.1) can be rewritten as:

$$
H=E_{g . s .}+\Delta E_{0}+\frac{1}{2 g} L^{2}+\delta L \cdot I+\gamma^{2}
$$

where $E_{g . s .}$ is the ground state energy, $\Delta E_{0}$ the band head energy and $g$ is the moment of inertia

$$
\begin{aligned}
E_{g . s .}= & \left(\sum_{K} \varepsilon_{K}(\beta) n_{K}\right)_{g . s .}+V_{p}(L=0)_{g . s .}+\left\langle H_{0}\left(n_{1}, n_{0}\right)\right\rangle_{g . s .}-B_{2}^{\pi v} C\left(n_{1}, 0\right) \\
\Delta E_{0}= & \Delta H_{\text {s.p. }}+\Delta\left(u_{1}, v_{0}\right)+\Delta H_{0}\left(n_{l}, n_{0}\right)+h \omega\left(n_{\gamma}+n_{\beta}+K / 2\right) \\
& \frac{1}{2 g}=\left[\frac{3}{8} B_{2}^{\pi N}+F\left(n_{1}\right)\right]
\end{aligned}
$$

From Eq. (2.10), one immediately recognizes that pairing will reduce the moment of netia, a well known nuclear structure effect now vividly seen. Also, one sees the microscopic meaning of stretching: pairing tends to increase $n_{I}$ as $L$ increases ( Eq. (2.6b)), which will increase the deformation $\beta$ and the moment of inertia $J$.

Since the FDSM is a microscopic PRM, it is not surprising that it contains the physics of high-spins. Thus far the $\mathrm{SU}_{3}$ limit of the model has been successfully applied to various aspects of low- and high-spin phenomena: for example, the study of ground state-deformation and the origin of a small window of oblate geometry in rare-earths, 22 the stretching and CAP (Coriolis anti-pairing) effects, 23 spin-alignment, 24 high-spin $B(E 2)$ 's, 18 and so on. In fact, the study of the basic features of most of these phenomena require only analytic and simple calculations, with transparent physical 
interpretations. However, one must be reminded that these are the zeroth order (symmetry limits), or the first order (perturbation) apprcximation of the model. With symmetry breaking terms such as the single-particle energy splitting, mixing due to pairing and Coriolis interactions and so on, further improvements are expected.

Let me now turn my attention to the primary subject matter of the talk: deformation in nuclear physics. Deformation is a macroscopic concept. Microscopically it can be defined either through the deformed mean field or the quadrupole moment :

$$
\begin{array}{cc}
\left\langle V_{\text {mean field }}\right\rangle_{\text {intrinsic frame }}=\beta m \omega_{0}^{2}\left\langle r^{2} Y_{20}\left(\theta^{\prime}\right)\right\rangle \\
Q_{d}=\frac{6}{\sqrt{5 \pi}} Z e R_{0}^{2} \beta \operatorname{Sin}\left(\gamma+30^{\circ}\right) & Q(L)=Q_{d} \frac{3 K^{2}-J(J+1)}{(2 J+3)(J+1)} \\
Q_{t}=\frac{6}{\sqrt{15 \pi}} Z e R_{0}^{2} \beta \operatorname{Cos}\left(\gamma+30^{\circ}\right), & B(E 2)=Q_{t}^{2} \frac{5}{16 \pi} / C_{J_{i} K_{i}}^{J_{f} K_{f}} /^{2}
\end{array}
$$

In Eq. (2.11), an axially-symmetric deformed field is assumed. Experimentally, one can extract deformation from Eqs. (2.12) and (2.13) by measuring the static quadrupole moment $Q_{d}$ and the transition quadrupole moment $Q_{t}$. Likewise, one can compute theoretical $Q(L ;$ and $B(E 2)$ values, and then deduce $\beta$ and $\gamma$. For the FDSM, by assuming the collective quadrupole operator to be

$$
Q_{2 \mu}=\frac{3 e}{4 \pi} Z R_{0}^{2} \chi P_{\mu}^{2}
$$

one can determine the deformation. For the $\mathrm{SU}_{3}$ symmetric and the $\mathrm{SO}_{6}(0,0,0)$ irreps, which correspond to an axially symmetric and a $\gamma$ soft rotor respectively,

$$
\begin{array}{ll}
\beta=\chi \sqrt{C\left(\lambda_{1} \mu\right)} \approx \chi n_{1} / \sqrt{2}, & n_{1}=2 \times \min \left(N_{1}, \Omega_{1}-N_{1}\right) \\
\beta=\chi \sqrt{\sigma(\sigma+4)} \approx \chi n_{1} / 2, & \sigma=\min \left(N_{1}, \Omega_{1}-N_{1}\right)
\end{array}
$$

For asymmetric $\mathrm{SU}_{3}$ irreps $(\mu \neq 0)$, it has been shown that the nuclear shape are triaxial $(\gamma \neq 0) .25$ Thus, within the FDSM context, the nuclear deformation can be microscopically understood as follows:

(1) the systematics $\beta$ are primarily determined by the particle (or hole) number $n_{1}$ in the normal-parity levels. These particles are responsible for the symmetries $\mathrm{SU}_{3}$ or $\mathrm{SO}_{6}$. For $\mathrm{SO}_{6}$, the maximum $\beta$ occurs at mid-shell of the normal-parity levels $\left(n_{1}=\Omega_{1}\right)$, where $\sigma$ is maximum $\left(\sigma=\Omega_{1} / 2\right)$. For $S U_{3}$, however, the maximum $\beta$ occurs when $n_{1}$ is equal to one third of the normal-parity shell degeneracy $\left(n_{l}=2 \Omega_{j} / 3\right)$, because that is where the maximum $\mathrm{SU}_{3}$ Casimir eigenvalue is found. Beyond that, deformation will begin to diminish until mid-shell for $n_{1}$, since $\left(n_{1}, 0\right)$ is Pauli forbidden (we call this the dynamical 
Pauli effect). The same behavior applies to the hole side of the shell, since $S U_{3}$ is particle-hole symmetric in $n_{l}$.

(2) The deformed geometry is also determined by $n_{l}$ and the dynamical symmetry . The geometry implied by the $S U_{3}$ symmetric irrep $\left(n_{1}, 0\right)$ can either be prolate or oblate, depending on whether $n_{1}$ is a particle or a hole number. It is interesting that when $n_{1}>$ $2 \Omega_{1} / 3,\left(n_{1}, 0\right)$ is forbiddeli and therefore the system is "Pauli-driven" to triaxial geometry $(\gamma \neq 0)$, while for $\mathrm{SO}_{6}$ it is always $\gamma$-soft.

(3) The particles occupying the abnormal-parity level do not directly contribute to deformation very much since they are dominated by $S$ pairs. For $S_{6}$, although the quadrupole coupling between the abnormal- and normal-parity levels via broken pairs can stabilize a $\gamma$ minimum and thus the system undergo a shape change from $\gamma$ soft to axial or triaxial shape. However, it will not alter much the value of $\beta .25$ For $S_{3}$ case, the abnormal-parity level serves mainly as a "sink" to accommodate nucleons "Pauli driven" out of the normal parity levels. The physical consequence is that nuclei can remain in the symmetric irrep, i.e. axial symmetry, in the physical mid-shell. It must be clearly stated that without the existence of an abnormal-parity level within the $S p_{6}$ shell, deformation will begin to decrease when the shell is one-third full, which means that no well-deformed nuclei could be found near the mid-physical shell! Thus the abnormalparity orbital pla; : an essential role in deformation, but that role is subtle one.

(4) If the $\mathrm{SO}_{6}$ symmetry is strongly coupled to an $S U_{3}$ symmetry through the quadrupole-quadrupole interaction, as in rare-earths, then recent numerical calculations have shown that the resultant ground band and their $B(E 2)$ 's do behave similarly to a pure $\mathrm{SU}_{3} 26$

There is an important question regarding the role played by the particles occupying the abnormal-parity level in nuclear deformation. This is so important in nuclear structure physics that it deserves further explanation. At first glance, the FDSM mechanism for deformation as outlined here seems to contradict the conventional wisdom, which states that nuclei are driven to deform by the down-sloping nature of the intruder orbitals (i.e. the abnormal-parity level) in the Nilsson scheme. Very recently, we have examined this point by using the standard Lund Nilsson-Strutinski code. Comparison was made of two calculations: one is the standard rare-earth deformation calculation with normally sloping intruder orbitals; the other is the same except the quadrupole coupling between the abnormal-parity level and the deformed field is artificially switched-off. In the latter, the abnormal-parity level becomes degenerate and deformation independent. The differences of these two calculations should unambiguously spell out the importance of down sloping intruder orbitals in the Nilsson systematics. Indeed, if the conventional wisdom is a correct view of deformation, then these two calculations should show quite different results. Yet, the difference turns out at most to be $20 \%$ for most nuclei! This implies that the Nilsson model is inherently 
consistent with the FDSM's fundamental assumption. Details of this investigation will be given in a forthcoming paper 27.

\section{THE SUPERSHELL FDSM (SFDSM)}

We are now ready to discuss the physics of superdeformation. Ever since the Liverpool group made the remarkable discovery of superdeformation (SD) for discrete states in ${ }^{152} \mathrm{Dy}$ in 1986 , its study has taken the center stage of high-spin physics. Theoretically, SD is still an enigma. On one hand it is sufficiently understood that the phenomenon were predicted a decade prior to the experimental discovery; on the other hand, there are many important and interesting systematics of SE, especially those explored recently (see Professor Twin's review in these proceedings), which I do not believe are fully understood.

There are currently at least four important physical questions concerning SD which need intuitive understanding. They are:

(1) Why does $S D$ appear only for nuclei in some regions of the periodic chart

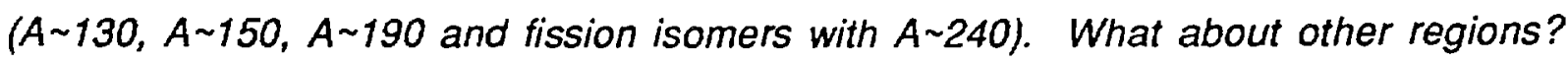
Although there have been relatively successful explanations of $S D$ as a consequence of shell-gaps 28,29 , one may still ask the deeper question: what causes such gaps to appear?

(2) Why does the g-ray intensity of a SD band decay out suddenly at a certain angular momentum, and why is the decaying out point different for different regions of SD nuclei. 28

(3) Why is it that the dynamical moment of inertia (2) behaves so differentiy for different regions of superdeformed nuclei: For A 190, all SD bands have similar values of $g(2)$, which typically increases smoothly as angular momentum (or frequency) increases. For A 150, however, $g^{(2)}$ mostly decreases with a great deal of variation from nucleus to nucleus. 28,29

(4) How does one understand the recently-observed identical (or "twinned") $S D$ bands, for example in ${ }^{151} \mathrm{~Tb}$ and ${ }^{152} \mathrm{Dy}$, and in ${ }^{150} \mathrm{Gd}$ and ${ }^{151} \mathrm{~Tb} .{ }^{30}$

One can immediately surmise that SD must be the result of the physics of multishells. Hence some fundamental differences with the physics of normal deformation must show up. There are two simple physical considerations for SD. One of them is the unusually large $f$ value, roughly twice as large as normal deformation $(0.2 \sim 0.3$ vs. $0.4 \sim$ 0.6). According to Eq. (2.15), this means that the number of participating nucleons for SD must be significantly larger than for normal deformation. In fact, this number can easily be estimated to be about 50 60 since the highest possible angular momentum $J$ of an SD band is approximately $60 \mathrm{~h}$ ( $30 \mathrm{D}$ pairs). On the other hand, as we have learned from normal deformation, the maximum deformation atiained by nuclei is when the active nucleon number reaches one third or one half of the shell degeneracy. To 
accommodate this physics, a large shell degeneracy is required. Clearly the usual onemajor-shell FDSM has neither a sufficient active nucleon number, nor large enough proton or neutron shull degeneracies, to sustain a SD.

Hence, in order to develop a theoret-

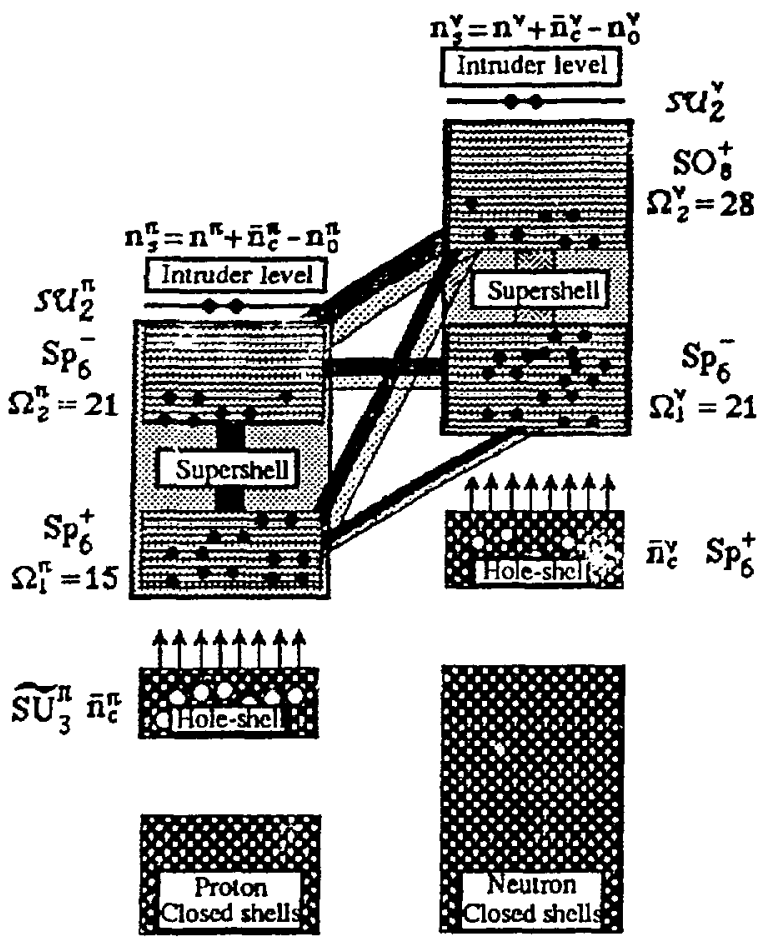

Fig. 4 A schematic picture of supershells for the $A \sim 150$ region.

The proton supershell has $S p_{6}^{+} \times S p_{6}^{-}$symmetry; while the neutron supershell has $\mathrm{Sp}_{6}^{-} \times \mathrm{SO}_{8}^{+}$ symmetry; " \pm "denotes the parity of the shell and $n^{\pi}$ and $n^{v}$ are the valence proton and neutron numbers; $\pi_{c} \pi$ and $\tilde{n}_{c}^{v}$ are the number of protonand neutron-holes in the hole-shells $n_{0}^{\pi}$ and $n_{0}^{v}$ are the number of protons and neutrons in the intruder level; $n_{s}^{\pi}$ and $n_{s}^{v}$ are the number of protons and neutrons in the supershells. ical model for SD within the FDSM framework, one must "locate" more active neutrons and protons and they must be allowed to "roam" among different major shells, say two at the minimum: one valence shell and a shell above it. We shall assume that the quadrupole-quadrupole correlation of the system in this physical scenario is sufficiently strong so that the usual shell gaps will not prevent particles to jump up. These stroncly correlated two shells are what we mean by a "supershell". However, while having more active shells can enlarge the shell degeneracies, it is not possible in this way to get additional active nucleons. To do so, the most economical way is to "dig below". This is why we have further relaxed the condition of shell closure and allowed additional nucleons to participate in the process of superdeforming the nucleus through the particle-hole excitation of the shell below the valence shell. Thus, in order to describe SD, three shells (neutrons and protons) are generally required. This idea is succinctly illustrated in Fig. 4.

It is also known that the quadrupole-quadrupole correlation strength between two shells is roughly proportional to the product of harmonic oscillator quantum numbers $\mathrm{N}$. This is because the expectation value of $r^{2}$ is proportional to $N$. Thus, the proton- and neutron-supershells are most strongly correlated with each other; this is illustrated in Fig. 4, where the bands between different shells depict the quadrupole-quadrupole correlation. An estimation shows that the coupling between the lower-shells ("holeshell" for short) and the supershells is about one order of magnitude weaker then the 
supershells themselves since among the three shells the hole-shells are the lowest h.o. shells, and generally contain the fewest active nucleons. Therefore we shall assume that it is the strongly coupled proton-neutron supershells that are primarily responsible for SD, while the hule-shells can be treated by a weak-coupling scheme. As we shall see, this is a crucial assumption for the model.

For $\mathrm{SD}$, since the system is more energetic than normal deformation, the picture is no longer just a single abnormal parity level "intruding" in a major shell. Rather, it is an entire upper shell strongly coupled to the valence shell. Thus, for SD, it is more convenient to classify shells according to the harmonic oscillator (h.o.) scheme. However, there is still an intruder level in the upper shell which cannot be ignored. For this level we shall assume the particles to be predominantly $S$ pairs, as in the normal FDSM.

The reclassification of h.o. shells in terms of $k-i$ quantum numbers and their symmetries in the $S$-D subspace is shown in Table 3 (shells with $N<3$ are not included).

Table 3. The Reclassification of Harmonic oscillator Shells*

\begin{tabular}{|c|c|c|c|c|c|}
\hline $\mathrm{N}$ & 3 & 4 & 5 & 6 & $?$ \\
\hline k & $\tilde{0} \tilde{2}$ & 1 & 11 & 15 & $\begin{array}{lll}1 & 1 & 1\end{array}$ \\
\hline i & $\frac{1}{2} \quad \frac{1}{2}$ & $\frac{1}{2} \quad \frac{7}{2}$ & $\frac{3}{2} \frac{9}{2}$ & $\begin{array}{ll}\frac{3}{2} & \frac{3}{2} \\
\end{array}$ & \begin{tabular}{|lll}
$\frac{1}{2}$ & $\frac{7}{2}$ & $\frac{13}{2}$ \\
\end{tabular} \\
\hline 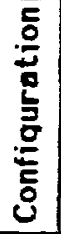 & $\begin{array}{r}P_{1 / 2} r_{3 / 2} \\
f_{3 / 2}\end{array}$ & $\begin{array}{ll}s_{1 / 2} & d_{5 / 2} \\
d_{3 / 2} & g_{7 / 2} \\
& g_{9 / 2}\end{array}$ & $\begin{array}{ll}p_{1 / 2} & f_{7 / 2} \\
p_{3 / 2} & h_{9 / 2} \\
f_{s / 2} & h_{1 / 2}\end{array}$ & $\begin{array}{ll}s_{1 / 2} & g_{7 / 2} \\
d_{3 / 2} & g_{g / 2} \\
d_{5 / 2} & i_{11 / 2} \\
& i_{5 / 2}\end{array}$ & $\left\{\begin{array}{rrr}P_{1 / 2} & f_{5 / 2} & h_{1 / 2} \\
P_{I / 2} & f_{1 / 2} & j_{13 / 2} \\
& h_{g / 2} & j_{15 / 2}\end{array}\right.$ \\
\hline 2 & 6 & 15 & 21 & 28 & 36 \\
\hline$n$ & 40 & 70 & 112 & 168 & 240 \\
\hline sym & $\widetilde{\mathrm{SU}_{3}}$ & $S p_{6}^{+}$ & $\mathrm{Sp}_{6}^{-}$ & $\mathrm{SO}_{8}^{+}$ & $\mathrm{Sp}_{6}^{-}$ \\
\hline
\end{tabular}

* In this table, $\mathbf{N}$ is the harmonic oscillator quantum number; $k$ and $i$ are the pseudn-orbital and pseudo-spin ; $\Omega$ is the pair degeneracy of the shell; $n$ denotes the total number of particles when the shell is completely filled. For shell 3 the pseudo harmonic oscillator and pseudo- $\mathrm{SU}_{3}$ symmetry is adopted.
For $N=3$ the pseudo- $S U_{3}$ $\left(\mathrm{SU}_{3}\right)$ symmetry is adopted. This is because with $N \leq 3$, there is no preference for seiecting either the $k$ - or $i$-active scheme, since for such shells woth $k \leq 1$ and $i \leq 3 / 2$. Indeed, if both coupling schemes are considered one obtains a closed algebra only for the entire shell, which suggests no further truncation for such shells. Although for the $N=3$ shell the $\mathrm{SU}_{3}(k) \times \mathrm{SO}_{6}(i)$ dynamical symmetry may be a candidate for rotations (see Table 2), we think that the pseudo-SU $\mathrm{SU}_{3}\left(\tilde{\mathrm{SU}}_{3}\right)$ symmetry provides a better truncation scheme since it is mathematically simpler and able to encompass a

larger configuration space. According to Table 3 , the SD configuration for a given nucleus is now determined. For example, for $A \sim 150$, the proton supershell consists of shells -4 and -5 with symmetry $S p_{6}^{+} \times S_{p_{6}}^{-}$, shell 3 with symmetry $\left(\widetilde{S U}_{3}\right)$ serves as the holeshell, and the intruder level $i_{13 / 2}$ comes from shell 6 . We will denote the configuration as

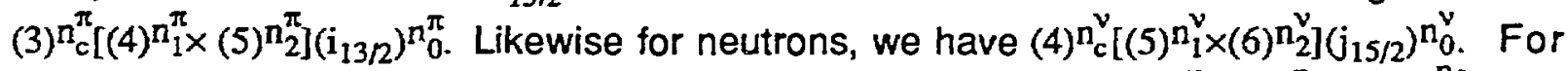
$A \sim 190$, both protons and neutrons will have configuration $(4)^{n_{c}}\left[(5)^{n_{1}} \times(6)^{n_{2}}\right]\left(j_{15 / 2}\right)^{n_{0}}$. 
Thus, there is a great deal of similarity between SFDSM for SD and the one-majorshell FDSM for normal deformation. In the latter, deformation is primarily a result of the normal-parity coherent $S-D$ pairs. The particle alignment, or in our language breakup of the $S$ pairs in the high-j intruder orbitals, plays a manifest role in high-spin physics. Likewise, in the SFDSM it is the coherent $S$-D pairs in the supershells which are responsible for superdeformation. There are also particles (dominated by $S$ pairs) which reside in the high-j intruder orbitals. They too can align and produce similar "high-spin" phenomena. In the one-major-shell FDSM, it is the $S_{3}$ limit

$$
S p_{6}^{\pi} \times S p_{6}^{v} \supset S U_{3}^{\pi} \times S U_{3}^{v} \supset S U_{3}^{\pi+v} \supset S O_{3}^{\pi+v}
$$

v:thich provides the explanation of many of the basic features of normal deformation. In SFDSM, there also is a $\mathrm{SU}_{3}$ dynamical symmetry:

$$
\begin{gathered}
\left(S p_{6}^{\pi 1} \times S p_{6}^{v 1}\right) \times\left(S p_{6}^{\pi 2} \times S p_{6}^{v 2}\right) \supset\left(S U_{3}^{\pi 1} \times S U_{3}^{v 1}\right) \times\left(S U_{3}^{\pi 2} \times S U_{3}^{v 2}\right) \supset \\
S U_{3}^{(\pi+v) 1} \times S U_{3}^{(\pi+v) 2} \supset S U_{3}^{\pi+v} \supset S O_{3}^{\pi+v}
\end{gathered}
$$

The indices $\pi l(v 1)$ and $\pi 2(v 2)$ denote the two h.o. shells in the proton (neutron) supershell. In the $\mathrm{SU}_{3}$ limit, $\beta$, the spectrum $E$, the two moments of inertia $x^{(1)}$ and $\mathfrak{x}^{(2)}$, etc can be computed by the same formulas as the one-major-shell case (Eq. (2.1)-Eq. (2.10) and (2.15)), except for the replacement of $\Omega_{1}$ and $n_{l}$ by $\Omega_{s}$ and $n_{s}$.

However, there are some important new features not present in the one-major-shell FDSM. For example, in the SFDSM, the parity of the intruder level is not unique. This is because both parities are present in the supershell. In fact, the term "abnormal parity" no longer applies here. This means that the interaction between the nucleons in the intruder orbit and the supershells generally must be much stronger than the normal deformed case. Another new ingredient is the existence of the weakly coupled holeshells. As we shall see, many SD features are intimately related to such features of the model.

\section{GENERIC FEATURES OF SUPERDEFORMATIONS}

In the previous section we have outlined the SFDSM and its $S U_{3}$ limit. Of course, nuclei do not have perfect symmetries. In reaistic calculations one must include the details of symmetry breaking effects, such as the splitting of single particle energies, the mixing of $\mathrm{SU}_{3}$ irreps due to pairing and so on. Here I would like to just show you the generic features of the $\mathrm{SU}_{3}$ limit, and see how well these features agree with the empirical evidence. Before I do so, I should mention a small complication. As we can see from Table 3 , the symmetry of shell 6 is not $S p_{6}$ but $S O_{8}$. Thus in the $n-p$ coupled scheme, the symmetry is not $\left(S U_{3}^{\pi} \times S U_{3}^{v}\right)$ but $\left(S U_{3}^{\pi} \times S O_{6}^{v}\right)$, and thus there is no exact $S U_{3}$ 
symmetry limit. However, as we have pointed out previously, numerical calculations by $\mathrm{Wu}$ and Vallieres do support an $\mathrm{SU}_{3}$-like synimetry for many properties of low-lying normally deformed rare-earth states where the symmetry is $\left(\mathrm{SU}_{3}^{v} \times \mathrm{SO}_{6}^{\pi}\right) .26$ Therefore, in the following we shall, as an approximation, assume that the $\mathrm{SU}_{3}$ energy formula Eq. (2.7) can be used for an $\left(\mathrm{SU}_{3} \times \mathrm{SO}_{6}\right)$ system and $\jmath, \delta$ and $\gamma$ are treated as parameters, except that there is no dynamical Pauli effect in the $\mathrm{SO}_{6}$ sector.

\subsection{The Location of Superdeformed Nuclei}

To seek where SD states should occur is equivalent to asking for what cc nbinations of $\mathrm{N}$ and $\mathrm{Z}$ is there a second minimum in energy in the $S U_{3}$ supershell configuration which has low enough excitation energy (roughly around $5 \mathrm{MeV}$ with respect to the normal ground band). The term supershell configuration means that the distribution of the $n_{s}$ particles in the valence and upper shells is roughly proportional to their shell degeneracies; i.e. $n_{2} / n_{1} \sim \Omega_{2} / \Omega_{1}$ where $n_{s}=n_{1}+n_{2}$, and 1 and 2 denote the valence and the upper shells respectively. In other words, the occupancy is uniformly spread over the two shells. An $\mathrm{SU}_{3}$ symmetry will correspond to superdeformed states with a supershell configuration.

Can an SD band from a supershell configuration occur in the right energy range? After all, SD formation requires exciting many nucleons from the valence to the upper shell, which naively should cost much more than $5 \mathrm{MeV}$. To see that this is feasible, let us consider the simplest example. Assume that we have a system of $n$ nucleons interacting via a quadrupole-quadrupole force of the form $-\chi p^{2} \cdot p^{2}$. The two shells of interest here have the following parameters: $\Omega_{1}=15$ and $\Omega_{2}=21$, and an energy gap between the two shells, $\varepsilon=4 \mathrm{MeV}$. I will define a normal deformed state as one where the $n$ nucleons occupy only shell 1 ; for the SD state, they are distributed in the two shells: $n_{1}=\left(\Omega_{1} / \Omega_{s}\right) n$ and $n_{2}=\left(\Omega_{2} / \Omega_{s}\right) n$ in shells 1 and 2 respectively. The supershell pair degeneracy for this example is $\Omega_{s}=\Omega_{1}+\Omega_{2}=36$. In the $S U_{3}$ limit, (assuming $L=0$ ), the energies for the normal deformed and superdeformed states are

$$
E_{g s}=-\chi C\left(\lambda_{1}, \mu_{1}\right) \text { and } E_{S D}=n_{2} \varepsilon-\chi C(\lambda, \mu)
$$

respectively. Then the SD state excitation energy is

$$
\Delta E_{S D}=n_{2} \varepsilon-\chi\left[C(\lambda, \mu)-C\left(\lambda_{1}, \mu_{1}\right)\right]
$$

where $\left(\lambda_{1}, \mu_{1}\right)$ and $(\lambda, \mu)$ are the $S U_{3}$ quantum numbers for shell 1 and the supershell respectively. When $n \leq 2 \Omega_{1} / 3$, say $i 0\left(n_{2}=\left(\Omega_{2} / \Omega_{s}\right) n \approx 6\right)$, the lowest energy symmetric irrep $(n, 0)$ is Pauli allowed. Therefore both $\left(\lambda_{1}, \mu_{1}\right)$ and $(\lambda, \mu)$ are $(n, 0)$, and $\Delta E_{S D}=6 \varepsilon=$ $24 \mathrm{MeV}$, which means that the SD states will occur high in energy. However, if $2 \Omega_{s} / 3 \geq$ $n>2 \Omega_{1} / 3$, say $n=24\left(n_{2}=14\right)$, then $\left(\lambda_{1}, \mu_{1}\right)=(n, 0)$ is Pauli forbidden. In this case, the $S U_{3}$ irrep of the normal deformation is $\left(\lambda_{1}, \mu_{1}\right)=(0,6)$, while $(\lambda, \mu)$ for the supershell is still 
$(n, 0)$ since $n \leq 2 \Omega_{s} / 3$. Thus, if the strength of $\chi$ is chosen reasonably, $0.17 \mathrm{MeV}$ say, then the energy of the SD state in this scenario will be

$$
\Delta E_{S D}=n_{2} \varepsilon-\chi\left[C(24,0)-C_{s}(0,6)\right]=14 \varepsilon-\chi[324-27]=56-297 \chi=5.5(\mathrm{MeV}) !
$$

This example clearly illustrates that it is the quadrupole-quadrupole correlation, entering fizre through the $\mathrm{SU}_{3}$ Casimirs, that allows the occurrence of SD states in the right E.lergy range. It also demonstratos the importance of the dynamical Pauli effect in "economizing" the energetics in forming SD: for $n$ too small $\left(\leq 2 \Omega_{1} / 3\right)$, no correlation energy is gained; for $n$ too large $\left(>2 \Omega_{s} \beta\right.$ ). the correlation energy gained is also too weak. Only when $n$ is in the vicinity of $2 \Omega_{s} / 3$ (or $4 \Omega_{s} / 3$ which corresfond to $2 \Omega_{s} / 3$ holes), will the system possess maximum quadrupole correlation energy, thereby allowing the SD to appear in the right energy range (in this example, 24 is exactly $\left.2 \Omega_{s} / 3\right)$.

Now let us consider the 3-shell case, (a supershell and a weakly coupled hole-shell). What are the favorable conditions to promote nucleons from the hole-shell to the supershell? It turns out that a similar and simple calculation can be made. The answer is as expected: the particle distribution required to maximize the correlation energy gain must be such that the particle numbers in the supershell, and the hole number $\bar{n}_{c}$ (i.e. $\left.2 \Omega_{c}-n_{c}\right)$ or particle number $n_{c}$ in the hole-shell, are $2 \Omega_{s} / 3$ and $2 \Omega_{c} / 3$ respectively $\left(\Omega_{c}\right.$ is the pair degeneracy of the hole-shell). The reason is again the dynamical Pauli effect.

Based on the above symmetry considerations, the most favorable neutron (proton) number $N(Z)$ for SD states to occur at low excitation energy can be written as follows:

$$
N(Z)=\left\{\begin{array}{l}
n_{\text {close }}+2 \Omega_{c} / 3+\left(n_{s}^{p p}, n_{s}^{h p}\right) \\
n_{\text {close }}+4 \Omega_{c} / 3+\left(n_{s}^{p p}, n_{s}^{h p}\right) \\
n_{\text {close }}+2 \Omega_{c}+\left(n_{s}^{p p}, n_{s}^{h p}, n_{s}^{h h^{h}}\right)
\end{array}\right.
$$

where

$$
n_{s}^{a a^{\prime}}=n_{1}^{a}+n_{2}^{a^{\prime}}(a=p, h) ; \quad n_{i}^{p}=2 \Omega_{i} / 3, n_{i}^{h}=4 \Omega_{i} / 3 ;
$$

and for $\mathrm{SO}_{8}$ shells, $\quad n_{i}^{p}=n_{i}^{h}=28$, except $n_{2}^{p}=n_{2}^{h} \approx 18$ if $n_{c} \neq 2 \Omega_{c}$.

For example, for shells 4-5-6, $n_{\text {close }}=40, \Omega_{c}=15, \Omega_{1}=21, \Omega_{2}=28$, one obtains $N(Z)=$ $82,92,96,106,112,126$. For shells 5-6-7, $n_{\text {close }}=70, \Omega_{c}=21, \Omega_{1}=28, \Omega_{2}=36$, and one obtains $N(Z)=136,150,154$, and so on. The reason why in the 3-shell scenario $\left(n_{c} \neq 2 \Omega_{c}\right.$ ), for the $\mathrm{SO}_{8}$ shell we have to assume $n_{2}$ to be 18 instead of 28 is because it would otherwise require too much single-particle excitation energy. For example, if the particle number is 92 , the configuration $(4)^{10}\left[(5)^{14}(6)^{28}\right]$ requires $48 \varepsilon$ single-particle excitation energy, while $(4)^{20}\left[(5)^{14}(6)^{18}\right]$ requires only $28 \varepsilon$. Although the maximum 
quadrupole correlation energy for $\mathrm{SO}_{8}$ is at mid-shell $\left(n_{2}=28\right)$, it is not likely that the difference can compensate a large energy of 20ع. A similar argument can be made about why $n_{s}^{\text {hh }}$ should be excluded as well when $n_{c} \neq 2 \Omega_{c}$. The $\tilde{S U}_{3}$ shell must be treated specially; the $2 \Omega / 3(4 \Omega / 3)$ rule cannot be applied, since the syinmetry is assumed to be psuedo- $\mathrm{SU}_{3}$ and not $\mathrm{Sp}_{6} \supset \mathrm{SU}_{3}$. The highest symmetric representation in this case is $(6,0)$, which corresponds to 6 holes in the $n=3$ shell (i. e., $n_{c}=6$ ).

Details of the favorable $N(Z)$

Table 4. The favorable $N$ and $Z$ numbers for superdeformed states

\begin{tabular}{|c|c|c|c|c|c|c|c|}
\hline \multicolumn{2}{|c|}{$\mathbf{N}$} & 0.2 & 3 & 4 & 5 & 6 & 7 \\
\hline \multicolumn{2}{|c|}{ Sym. $\quad 2 \Omega$} & 28 & 12 & 30 & 42 & 56 & 72 \\
\hline$n_{c}\left(\bar{n}_{c}\right)$ & $n$ & $\begin{array}{l}\text { Cloped } \\
\text { Sheell }\end{array}$ & $\tilde{S U}_{3}$ & $\mathrm{Sp}_{6}^{+}$ & $\mathrm{Sp}_{6}^{-}$ & $\mathrm{SO}_{\mathrm{s}}^{+}$ & $\mathrm{SP}_{6}^{+}$ \\
\hline 6 & 58 & (28) & 6 & 10 & 14 & & \\
\hline$\dot{0}$ & 64 & & (40) & 10 & 14 & & \\
\hline$\overline{\mathbf{0}}$ & 74 & & (40) & 20 & 14 & & \\
\hline$\dot{0}$ & 88 & & (40) & 20 & 28 & & \\
\hline 10 & 82 & & (40) & 10 & 14 & 18 & \\
\hline$\overline{10}$ & 92 & & (40) & 20 & 14 & 18 & \\
\hline 10 & 96 & & (40) & 10 & 28 & 18 & \\
\hline$\overline{\mathbf{1 0}}$ & 106 & & (40) & 20 & 28 & 18 & \\
\hline 0 & 112 & & & (70) & 14 & 28 & \\
\hline 6 & 126 & & & (50) & 28 & 28 & \\
\hline 14 & 136 & & & (70) & 14 & 28 & 24 \\
\hline$\overline{14}$ & 150 & & & (70) & 28 & 28 & 24 \\
\hline$\overline{0}$ & 164 & & & & (112) & 28 & 24 \\
\hline
\end{tabular}

number for $S D$ calculated by Eq.(4.4) are given in Table 4, and a map locating of favored regions for SD nuclei is presented in Fig. 5. In the map the hatched regions represent the possible SD regions. For two shell configurations $\left(\bar{n}_{c}=0\right)$, the range is determined by allowing an uncertainty of \pm 1 pair. For example, according to table 4, 74 is one of the favorable $N(Z)$ numbers, the configuration is $(3)^{\overline{0}}\left[(4)^{20}(5)^{14}\right]$ and the possible range is $72-76$. For 3-shell configurations the range is determined by allowing $n_{c}$ to vary from $2 \Omega_{d} / 3$ to $4 \Omega_{d} / 3$, with an one pair uncertainty. For

example, in Table 4, 82 and 92 are predicted to be two favorable $N(Z)$ number; their configurations are $(3)^{10}\left[(4)^{14}(5)^{18}\right]$ and $(3)^{20}\left[(4)^{14}(5)^{18}\right]$ respectively. We assume the range for SD to possibly occur is $80-94$. The reason for this is that on one hand, they all have the same supershell structure, which is mostly responsible for the super deformation; on the other hand, in this range of $n_{c}$ the variation of the quadrupole correlation in the hole shell is the smallest, provided the quadrupole correlation itself in the hole shell is already the weakest. Thus the excitation energy of SD states should be quite close for this range of particle numbers, and therefore all are possible. The same arguments apply for the ranges $94-108$ and 134-152. Chasman has calculated superdeformation for nuclei near $A \sim 190.31$ He predicted a SD region $67<Z<78$ and $96<\mathrm{N}<106$. However, for most of them the SD minimum is shallow. He suggested that ${ }^{178} \mathrm{~W}$ might be the most likely SD nucleus in this region. This prediction is consistent with the region $Z \sim 74$ and $94<N<108$ predicted by our model (see Fig. 5). 


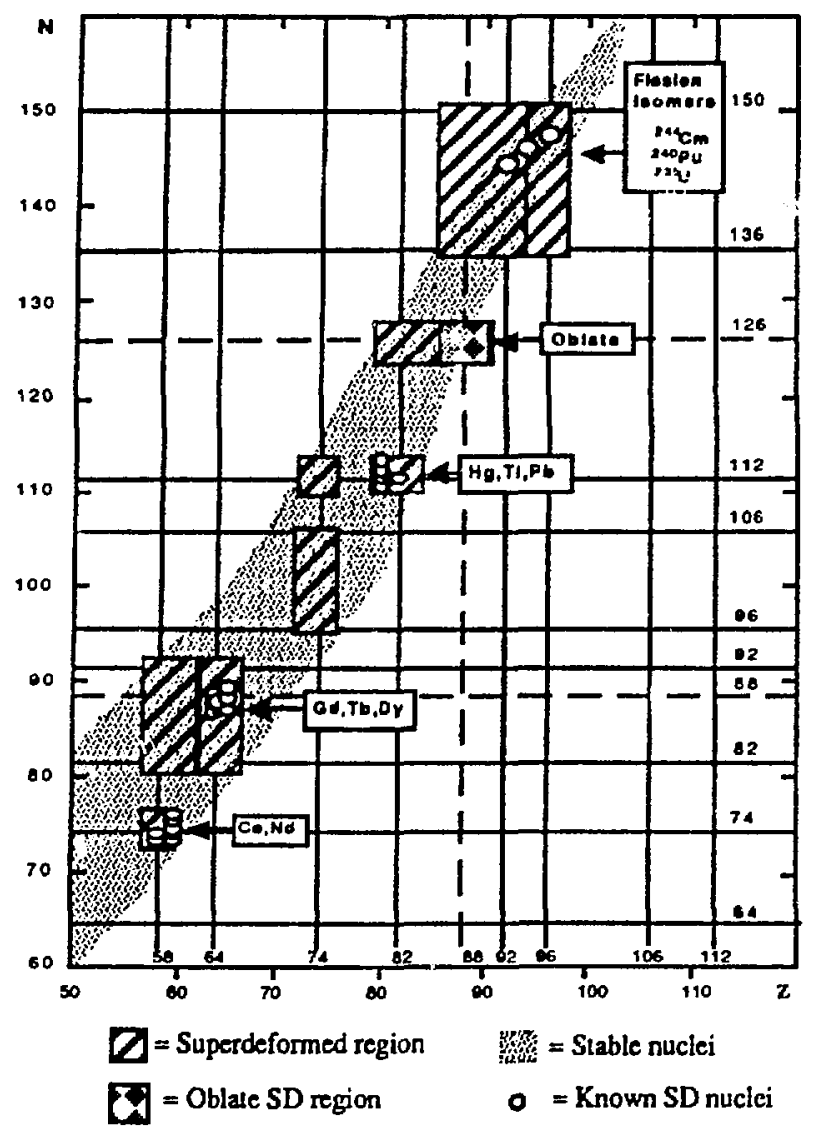

Fig. 5 The SFDSM predicted map of superdeformed nuclei

Of course, one should only expect Fig. 5 to be qualitatively accurate since many details like single-particle energy splitting, Coulomb interaction, pairing interaction, angular momentum effects, and so on, have not been considered. The regions predicted are just the possible regions. In each region, there may be some fine structure; not every nucleus in these regions are guaranteed to be SD nuclei. To compute precisely the SD excitation energy and its actual configuration for a specific nucleus, a more detailed calculation is required. Still it is remarkable that the global SD map is well reproduced by such a simple symmetry consideration!

At this point I would like to bring out a very interesting relationship between the Nilsson shell gaps and the FDSM. There are two famous Nilsson shell

gaps. 98 and 152 nucleons in the82-126 and 126-184 shells respectively. I would like to stress that the occurrence of these shell gaps is due to the dynamical Pauli effect of the $\mathrm{FDSM} \mathrm{SU}_{3}$ symmetry. This can be shown as follows: using the FDSM semi-empirical formula $n_{l}=1.5+n / 2$, one sees that 98 nucleons $\left(n=16\right.$ ) corresponds to $n_{1} \approx 10$, which is precisely $2 \Omega_{J} / 3$ for the $82-126$ shell and 152 nucleons corresponds to $n_{l} \approx 14$, which is $2 \Omega_{l} / 3$ for the $126-184$ she". Beyond these points, to accommodate an $S_{3}$ irrep change the system must experience an energy jump. In mean-field language, this is precisely a shell gap! Obviously, a similar situation now exists in the SD case as well. Traditionally, the locations of SD nuclei are "determined" by some large Nilsson shell gaps. What we have shown here is that the shell gaps themselves are related to the dynamical Pauli effects of the supershells.

In the $S_{3}$ limit the geometry of a SD nuclei is determined by its supershell irrep $(\lambda, \mu)$ : if the nucleon number in the supershell satisfies $n_{s} \leq 2 \Omega_{s} / 3$, it is prolate; if $n_{s} \geq 4 \Omega_{s} / 3$ 
(hole configuration), it is oblate; if $n_{s}$ is between $2 \Omega_{s} / 3$ and $4 \Omega_{s} / 3$, it will either be triaxial or $\gamma$ soft. Thus, from Table 4 and Fig. 5 one sees that most SD nuclei are prolate. This too is apparently in agreement with existing data. Indeed, we find that the hole configuration for a supershell requires more particle excitations, and thus usually is energetically unfavorable. Still, there is a region where oblate shape SD nuclei might occur which is $(N, Z)=(126,88)$ (see Fig. 5). It would be of great interest to see whether such SD states can be observed.

\subsection{The decaying out point for the E2-transitions in the SD bands}

A puzzling aspect of SD is how and why the intensity decays so suddenly from a superdeformed band. There appears to be no simple and satisfactory urderstanding of this phenomena. In fact the decaying out point for different mass regions are distinctly different. For example, for mass 190 , it is $\sim 8 h$ and for 150 , it is $\sim 20 h$. Prior to the decaying out, the $\gamma$ ray intensity within the yrast SD band is virtually a constant. After that, it rapidly diminishes within one or two steps. I will show in this section that this is a natural consequence of the weakly coupled hole-shell phenomena. It is closely related to the hole-shell D pair alignment (designated simply as "Q-pair-alignment (DPA)" from now on).

In standard high-spin nomenclature, band crossing usually means the rotational alignment of high-j neutrons or protons (high-j is another terminology for the intruder orbital). Depending on whether the band interaction is strong or weak, this crossing will cause the moment of inertia to up- or back-band. At lower spins a smooth alteration of the moment of inertia (VMI) is a result of the so-called CAP (Coriolis antipairing) and stretching effects. Such effects are usually accommodated phenomenologically. From the FDSM point of view, CAP is also a band crossing effect due to the alignment of the pseudo-spin (i) of a pair (i.e. broken pair) in the normal-parity levels. Unlike those in the intruder level, a pair breaking in the normal-parity levels is a collective phenomenon and will spread its effect over all the relevant orbitals, hence the term CAP. The CAP band crossing occurs at relatively low spins and there is a strong interaction with the ground band; this smoothly increases the moment of inertia, which together with the stretching effect is precisely the phenomenology of the VMI effect

Because of additional ingredients, the physics of SD from the SFDSM is more intriguing than that of normal deformation. Not only are there high-j and CAP band crossings; there are also the S-D pairs in the hole-shell which are weakly coupled to the superdeformed core (the supershell). Physically, when a nucleus starts to rotate the hole-shell D-pairs can align at no cost in energy (because no pairs must be broken), and therefore this should happen first. The effective moment of inertia of such a DPA band is always larger then the unaligned SD band. In the usual high spin physics an unaligned band should be the ground band; here it is not. The DPA band is now the SD ground band. At higher frequencies the CAP bands will cross the DPA band, followed 
by the crossing of a high-j neutron or proton aligned band. Beyond the crossing point of the DPA and CAP bands the yrast SD band is formed by mixing the CAP and high- $j$ aligned bands. This is what we shall call the high-spin SD yrast band.

As we have emphasized before, the intruder orbitals in the SD case are no longer unique-parity levels. This allows strong interaction between the CAP and high-j aligned bands, and implies that for SD the band crossing between CAP and high- $j$ aligned bands will usually be smooth and not be expected to come a "back bend". This feature is consistent with the smooth $\not 1$ (1) behavior of most SD nuclei.

With this general SD band crossing scenario the decaying out mechanism can now be understood as follows: the decaying out point is just the crossing point between the DPA band (low-spin SD yrast band) and the high-spin SD yrast band, as I will explain in more detail below.

iypical SFDSM calculations for ${ }^{194} \mathrm{Hg}$ and ${ }^{150} \mathrm{Gd}$ are presented in Fig. 6. These calculations use the $\mathrm{SU}_{3}$ formula of Eq.(2.7). The parameters g, $\Delta, \delta$ and $\gamma$ for each band are determined by fitting the $\gamma$ transitional energies. The mixing of the CAP, neutron and proton high-j bands are all considered with interaction matrix elements between bands assumed to be a constant of $0.2 \mathrm{MeV}$. However, as we shall see, such details are not crucial for understanding the decaying out mechanism. What governs the physics is the weak coupling (thus sharp crossing) between the DPA and the supershell bands, and that the DPA band is actually an envelope of SD bands with different D-pair alignment in the hole shell. At frequencies larger than the crossing point there are no superdeformed states below the SD yrast band. Thus, when the decay comes downward from high spins the transition can only proceed along the yras, line. This explains the constant the intensity. However, as soon as the crossing point is reached the DPA band is now yrast and is weakly couple to the CAP and high-j aligned bands; thus, the decay can no longer proceed along the yrast line. Instead, a fragmentation of the $\gamma$ transition will occur since there are now many SD states lying between the DPA band and the extension of the high-spin yrast band. This is the explanation offered by the SFDSM for the sudden drop in $\gamma$-ray intensity (see Fig.7).

It should be noted that there are actually two sequential processes involved in the $S D$ decaying out problem: one is the decaying out from the yrast $S D$ band to non-yrast $S D$ bands, characterized by different D-pair alignment (the process from $(a)$ to $(b)$ in Fig. 7); the other is the decaying out from the $S D$ bands to the normal deformed bands (the process from $(b)$ to $(c)$ in Fig. 7). In the first process, the strength remains in SD states, but is fragmented into so many SD bands that discrete superdeformed transition are no longer seen. This is what experimentally found and, as we have just discussed, the SFDSM provides a mechanism causing this sudden loss of intensity in the yrast SD band. This population, of course, must then decay to the normal deformed states, but that is a separate process. We do not have much information about this process yet. 

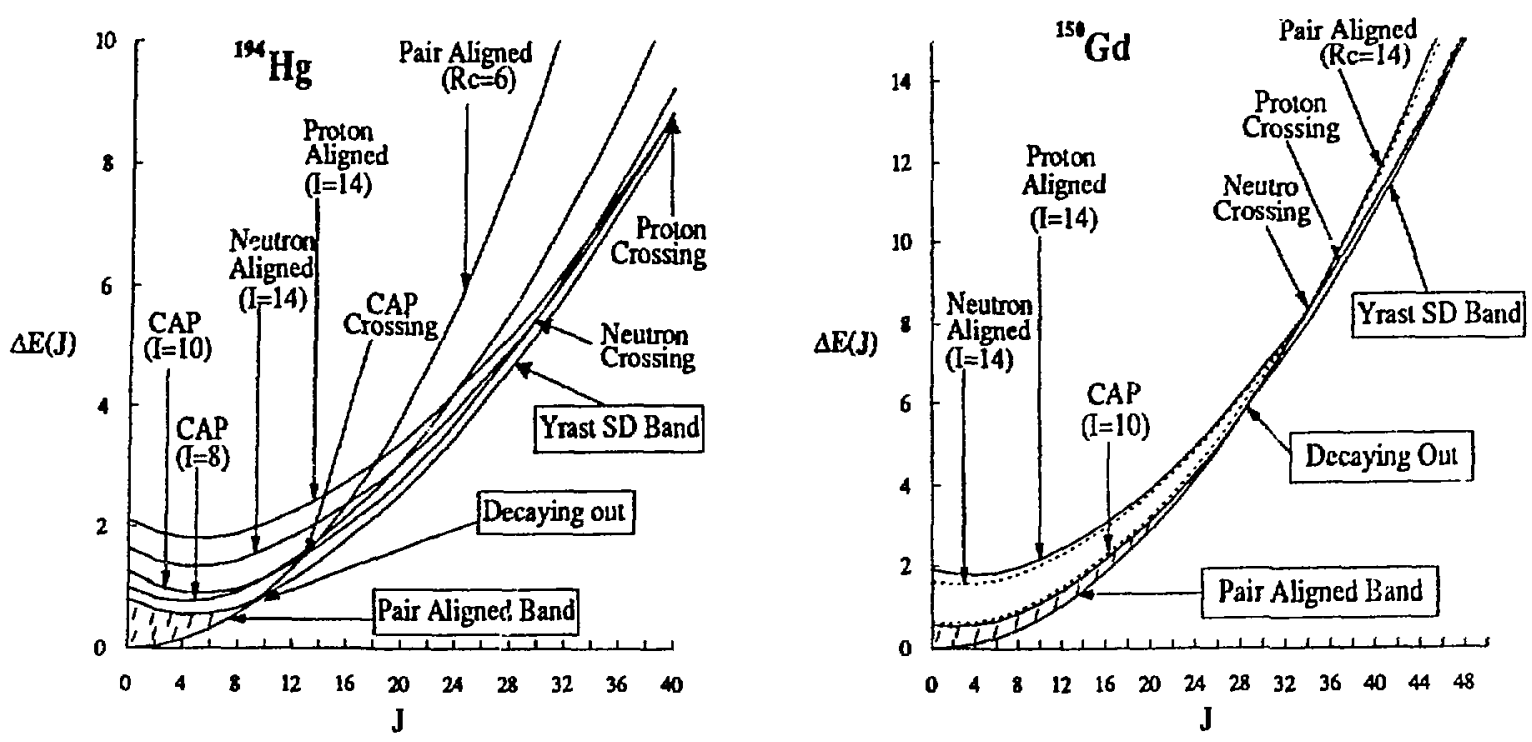

Fig. 6 The FDSM calculations for SD band crossings and the decaying-out point.

In the figure, the maximum alignment for each band, $I=2 i-1$ for $S_{p 6}$ shells, and $2 k$ for $S_{8}$ shells, are presented. The maximum alignment for pairs in the hole-shell is $R_{C}=n_{C}$. For ${ }^{194} \mathrm{Hg}$, two CAP bands are considered: one in shell $5(i=9 / 2), I=8$; the other in shell $6(k=5), I=10$. The high-j protons and neutrons are both in the $j_{15 / 2}$ orbit, $I=14$, and $R_{c}=6$. For ${ }^{150} \mathrm{Gd}$, only one CAP band is considered (shell $6, k=5$ ), $I=10$. High-j protons are in the $i_{13 / 2}$ orbit, $I=12$, and neutrons are in the $j_{15 / 2}$ orbit, $I=14$, and $R_{C}=14$. In both cases the yrast band is obtained by mixing CAP and high-j aligned bands. The hatched regions indicate where the fragmentation of $\gamma$-intensity takes place. The decaying out point is predicted to be $J=10$ for ${ }^{194} \mathrm{Hg}$ and $J=28$ for ${ }^{150} \mathrm{Gd}$.

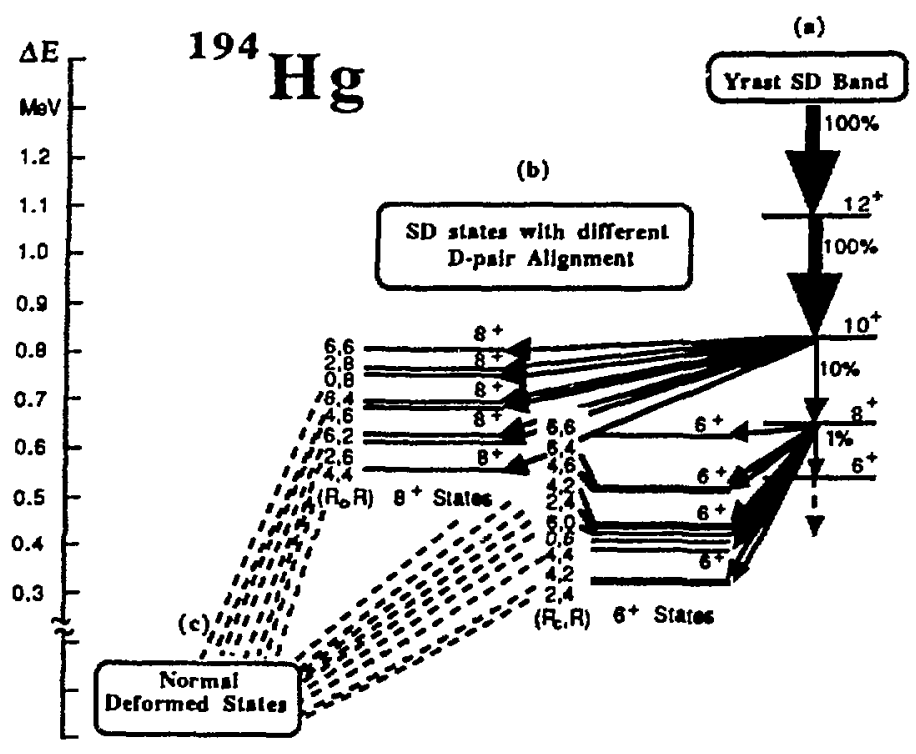

Fig. 7 The Fragmentation of $\gamma$ Intensity in the Superdeformed Band of ${ }^{194} \mathrm{Hg}$ Notice that the fragmentation causing the loss of $\gamma$ intensity occurs only between superdeformed states. 
It is noteworthy that the crossing point depends sensitively on the maximum D-pair alignment in the hole-shell. Its value is equal to the number of holes $\bar{n}_{c}$ (or particles $n_{c}$ ). Naturally, the larger the $n_{c}$ the higher the crossing angular momentum $J_{c}$. It can be shown that for $A \sim 190, n_{c}$ is about 6 while for $A \sim 150 n_{c}$ is about 14 . For example, the SD configurations for ${ }^{194} \mathrm{Hg}$ and ${ }^{150} \mathrm{Gd}$ are:

$$
\begin{aligned}
{ }^{194} \mathrm{Hg}: & \pi(4)^{6}\left[(5)^{14}(6)^{18}\right]\left(\mathrm{j}_{15 / 2}\right)^{2} & { }^{150} \mathrm{Gd}: & \pi(3)^{0}\left[(4)^{19}(5)^{12}\right]\left(\mathrm{i}_{13 / 2}\right)^{2} \\
& v(4)^{0}\left[(5)^{14}(6)^{26}\right]\left(\mathrm{j}_{15 / 2}\right)^{4} & & v(4)^{14}\left[(5)^{14}(6)^{16}\right]\left(\mathrm{j}_{15 / 2}\right)^{2}
\end{aligned}
$$

This explains why the decaying out point is different in different $\mathrm{SD}$ regions.

There is an uncertainty inherent in assigning the SD configuration. It is the unknown nucleon number in the intruder level $n_{o}$, which has not been taken into account in Table 4. However this uncertainty is not expected to alter the conclusions since $n_{o}$ can noi be a large number. The reason is that the intruder level is located in the upper part of the shell; hence to promote particles to that level would require a large single-particle excitation energy. Also, in SD the pairing energy is small and will be dominated by the strong quadrupole correlations. Therefore, the nucleon number distribution is primarily determined by the competition between the quadrupole correlation and the energy required to excite particles across the spherical shell gaps. If the intruder level is taken into account, the number listed in Table 4 for the upper-shell may be interpreted as $\left(n_{1}+n_{0}\right)$ with a one or two pair uncertainty.

\subsection{The Moment of inertia}

As Professor Twin has emphasized, the moments of inertia, $g(1)$ and $g(2)$ have become crucial physical quantities for SD investigations. Theoretically, the cranking shell model has reasonably reproduced these data (see refs. 28 and 29, and Twin's review in these proceedings). We can now compute these quantities as well. We have calculated a series of SD nuclei for $\mathrm{Hg}$, Dy and $\mathrm{Gd}$ isotopes. Two typical examples $\left({ }^{194} \mathrm{Hg}\right.$ and $\left.{ }^{150} \mathrm{Gd}\right)$ are presented in Fig. 8. Because of the time constraint I will not present the details here (they will be presented in a forthcoming article), nor will I dwell $c$ the excellent agreement. My primary concern here is the generic features, for which the details are really not crucial. What stands out in Fig. 8 is that $g^{(2)}$ for ${ }^{194} \mathrm{Hg}$ and ${ }^{150} \mathrm{Gd}$ are very different; furthermore these difference are typical for these two mass regions. ${ }^{194} \mathrm{Hg}$ (or other examples in the mass 190 region), $x^{22}$ generally increases with $\omega$ while for ${ }^{150} G d$ it decreases. On the other hand $\not 1$ ), with very few exceptions, it is generally either flat or increasing slightly in both regions, and $w ?$ do not observe :any back- or up-bending. I will now explain this generic behavior.

To this end, let's first recapitulate some basic physical facts about two crossing bands with a band-band interaction $v_{12}$. For simplicity, let us assume that the moments of inertia $g_{1}$ and $g_{2}$ of the two bands, the excitation energies $\Delta_{1}$ and $\Delta_{2}$, and the 


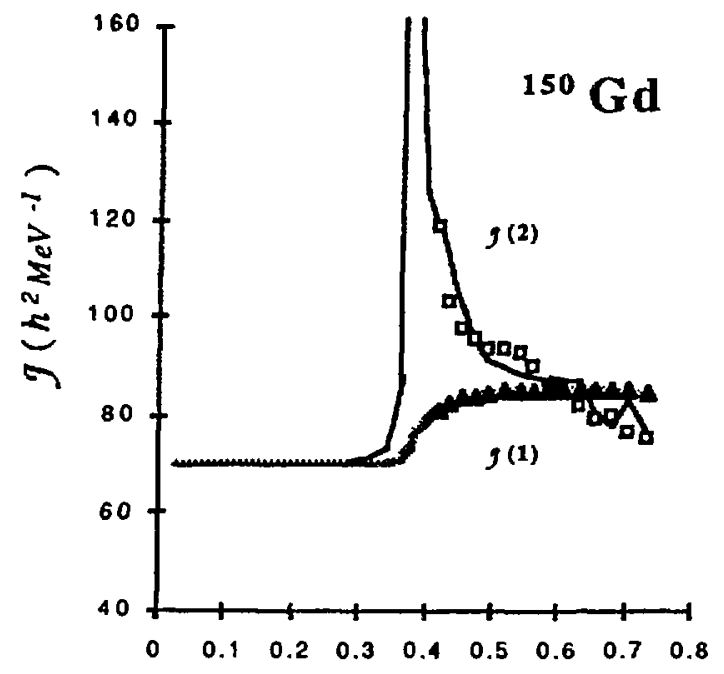

()

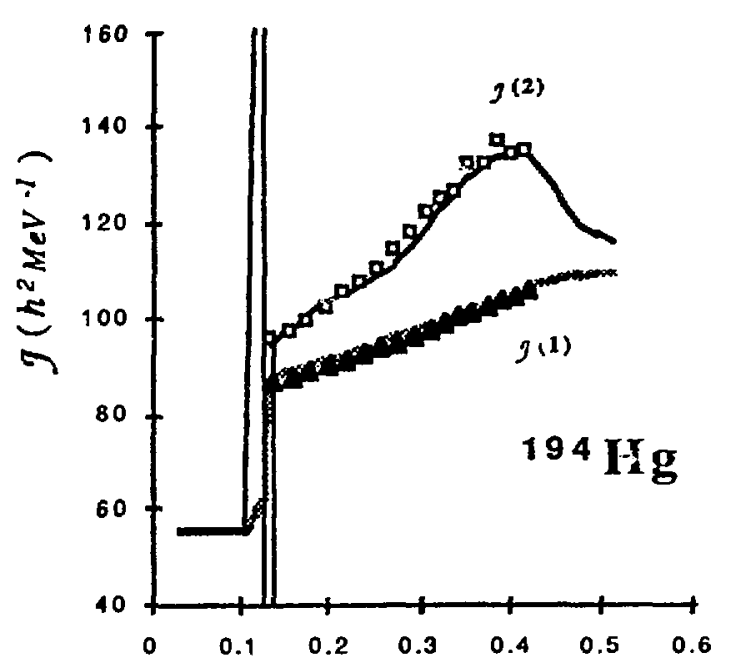

$\boldsymbol{\omega}$

Fig. 8 The moment of inertia of ${ }^{194} \mathrm{Hg}$ and ${ }^{150} \mathrm{Gd}$

In this figure, the dots are the data and lines are the SFDSM predictions.

Data are taken from refs. 31 and 32.

interaction $v_{12}$ are all constants. The calculation is then trivial and the results are displayed on the I.h.s. of Fig. 9. They show that if $V_{12}$ vanishes (Fig. 9 (a)), $x^{2}$ ) must be a step function with value $\jmath_{1}$ below $\omega_{c}$, and $\jmath_{2}$ above. Also, except for the singularity at $\omega_{c}, g(2)=g(1)$ (Fig. $9($ a)). Of course, a small interaction will remove the singularity and $\not 2)$ will increase significantly when $\omega$ approaches $\omega_{c}$ from either side; in this case $\not x^{(1)}$ will exhibit a back- or up-bend (Fig.9b). The nearly singular behavior of $g(2)$ is washed out for sufficiently large $v_{12}$, as is shown in Fig. 9c; $\not(2)$ becomes smooth with a bump appearing at $\omega_{s}$ (which slightly larger then $\omega_{c}$ ), and approaches a constant $l_{2}$ for a sufficiently large frequency.

Let us now investigate a 3-band crossing scenario: here we shall assume that there are two strongly coupled excited bands with moments of inertia $y_{1}$ and $y_{2}$, as in Fig. 9 (c), and a ground band with moment of inertia $\jmath_{0}$ which is weakly coupled to these bands. A schematic diagram is given in Fig. 10, and typical results are displayed in Fig. 9 (d)-(f). Comparisons of Figs. 9 (d) and (f) and Fig. 8 immediately suggest that the behavior of ${ }^{194} \mathrm{Hg}$ resembles that of Fig. 9 (d), while ${ }^{150} \mathrm{Gd}$ resembles that of Fig. 9 (f).

Of course, such a scenario is not a proper simulation for normal deformation since, as we have mentioned, the ground band interacts very strongly with the CAP bands. However, in the SD case, the ground band, as we have emphasized, is the DPA band, which is supposed to be weakly coupled to the excited bands. Hence, such a simple 3band picture may be a reasonable simulation of the SFDSM. As a matter of fact, one can actually derive a 3-band model from the SFDSM: the ground DPA band, and two 

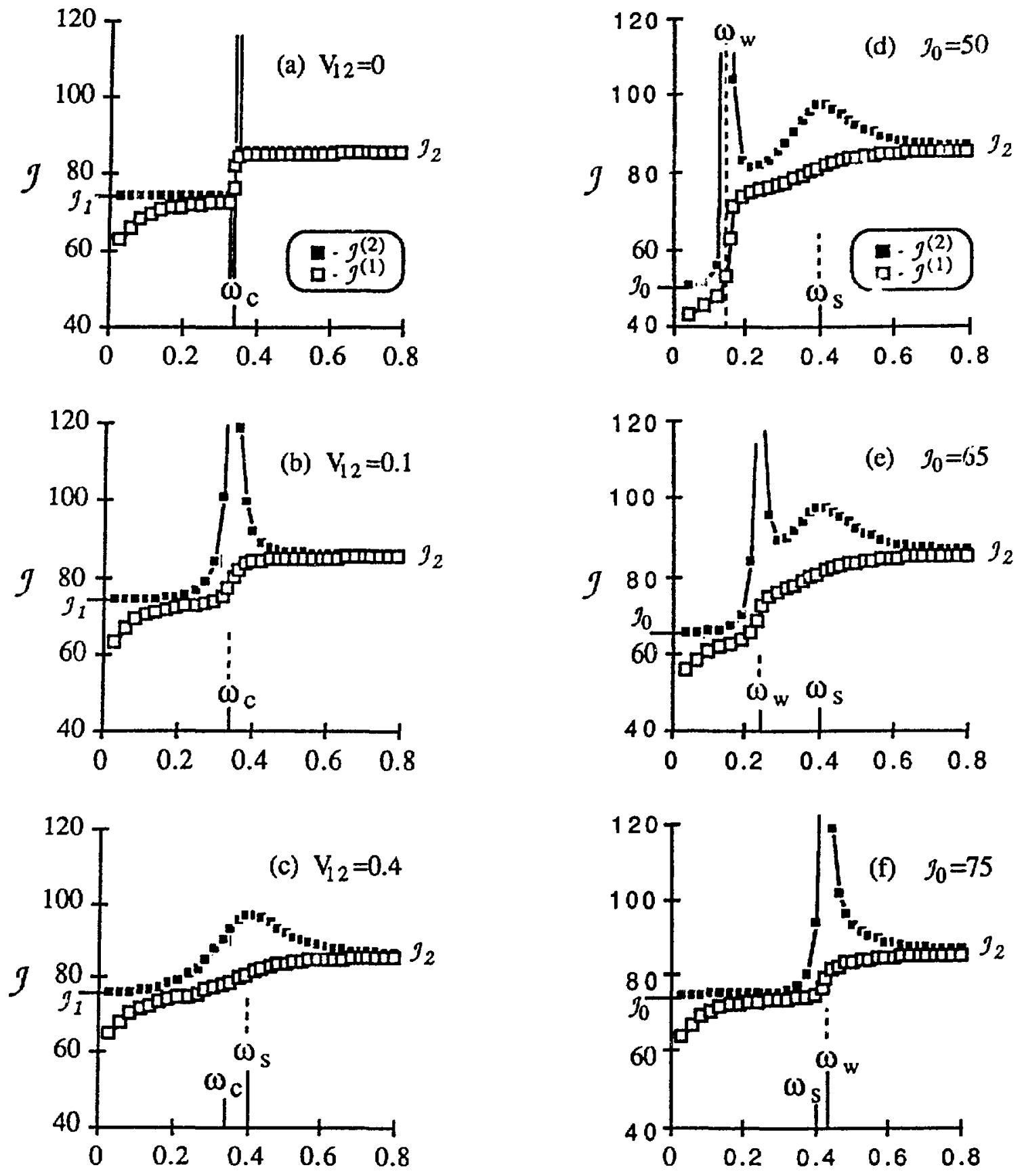

$\omega$

$\omega$

Fig. 9. The general behavior of the moments of inertia $\not^{22}$ and $\not \not^{11)}$.

In (a)-(c), the moments of inerlia of a 2-band crossing scenario with different band interactions $V_{12}$ are presented. In the plots, $\omega_{c}$ is the crossing frequency of the two bands; $\omega_{s}$ is the peak frequency of the $\left.x^{2}\right)$ curve when the interaction is strong. When the interaction is weak, $\omega_{s} \approx \omega_{c}$. In (d)-(f), the moments of inertia of a 3 band crossing scenario are presented: two excited bands as in case (c) plus one ground band weakly coupled to the excited bands. The parameters are: $g_{1}=$ $74, y_{2}=86, \Delta_{1}=0.5, \Delta_{2}=1.2$. The ground band moments of inertia $y_{0}$ are indicated in the figures . The interactions between the ground and excited bands are assumed to be small. For all these plots, a small interaction $V=0.05$ is used to removed the singularity at the crossing poi:it. 
excited bands which are a mixed CAP band and a high- $j$ aligned band, where the mixed CAP band is the CAP band strongly mixed with the unaligned band. The moments of inertia $g_{0}, g_{1}$ and $g_{2}$ are all effective ones, and are functions of the microscopic parameters of the SFDSM Hamiltonian. It can be shown that these moments of inertia can approximately be regarded as constants until the alignments reach their maximum values, beyond which they must be reduced. This effect of maximum alignment is important to explain why $g^{(2)}$ can become smaller then $\mathcal{X}^{1}$ ) at a certain high frequency (see Fig. $8,{ }^{150} \mathrm{Gd}$ data). Such a detailed feature is beyond the scope of the simple 3 band scenario (see Fig. 9 (f)). Still, we see that our simple picture has captured the essence of the SD physics.

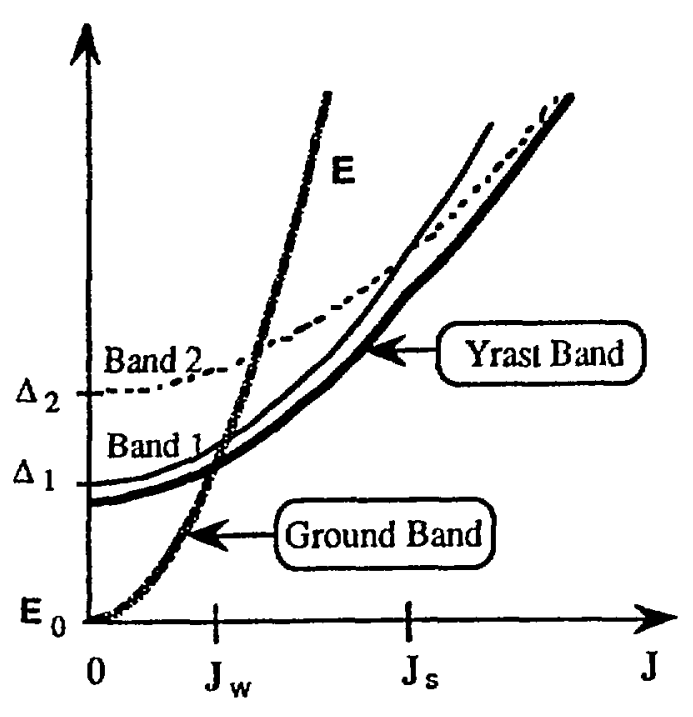

Fig. 10 A schematic representation of a three band scenario

The ground band here represents the DPA band. Band 1 represents the mixed CAP band, and band 2 represents the high-j band. In this scenario, band 1 and band 2 are strongly mixed, while mixing is weak between the ground band and bands 1 and 2.

From this study, it is clear that the pattern for $x^{(2)}$ is controlled by the sharp crossing point of the DPA band. In fact, the 3-band scenario can be viewed as a combination of two physical pictures: a strong mixing picture at high frequency (Fig. 9 (c)), and a sharp band crossing picture at low frequency (Fig. 9 (b)). If the crossing frequency $\omega_{w}$ is low, the pattern is that in Fig. $9(\mathrm{~d})$; for a higher crossing point where the sharp crossing nearly coincides with the position of the $g(2)$ bump $\omega_{s}$, the pattern will be given by Fig. $9(f)$; Fig. $9(e)$ is the intermediate situation. For $A \sim 190$, the crossing frequency $\omega_{w}$ is low because $n_{c}$ is small (see Table 4; e.g., $n_{c}=6$ for ${ }^{194} \mathrm{Hg}$ ). Also, for high frequency fission takes place, thereby cutting off the part of the curve for $x^{(2)}$ beyond the maximum of the bump. Hence, SD nuclei in the mass 190 region all behave as the rising part of the bump of $x^{(2)}$ (see Fig. 9(d)). For $A \sim 150, n_{c}$ is much larger (14 for ${ }^{150} \mathrm{Gd}$ ), thus the crossing point is moved up. It happens that $\omega_{w} \sim \omega_{s}$. This will significantly alter the strong mixing picture of the two excited bands. Hence, for this mass region the pattern is like Fig. 9 ( $f$ ). In fact, because of the dominance of the sharp crossing the resultant $x^{(2)}$ is very much like that of the two sharp crossing band situation of Fig. 9 (b).

For $g(1)$, as indicated in Fig. 9, one expects to observe a back-bend or up-bend around the crossing frequency, where $g(2)$ has a sharp peak. Unfortunately, this experimental situation cannot be realized since it occurs below the decaying out point, which is precisely the sharp peak point of $g(2)$. Hence, what one observes 
experimentally is a "truncated curve" (see Fig. 8). This explains why $\mathfrak{x}^{(1)}$ nearly always appears to be smooth (see Fig. 9).

It should be pointed out that what we discuss here are the general features expected for $g^{(1)}$ and $g(2)$. This does not exclude that for some particular high-j orbitals the interaction matrix elements between the intruder level and the mixed CAP band might be small, and thus some sharp crossing features may occur. The recently found $193 \mathrm{Hg}$ excited band and the ${ }^{146} G d$ yrast SD band may, correspond to this situation. These special cases are beyond the dynamical symmetry limit and require a more detailed calculation.
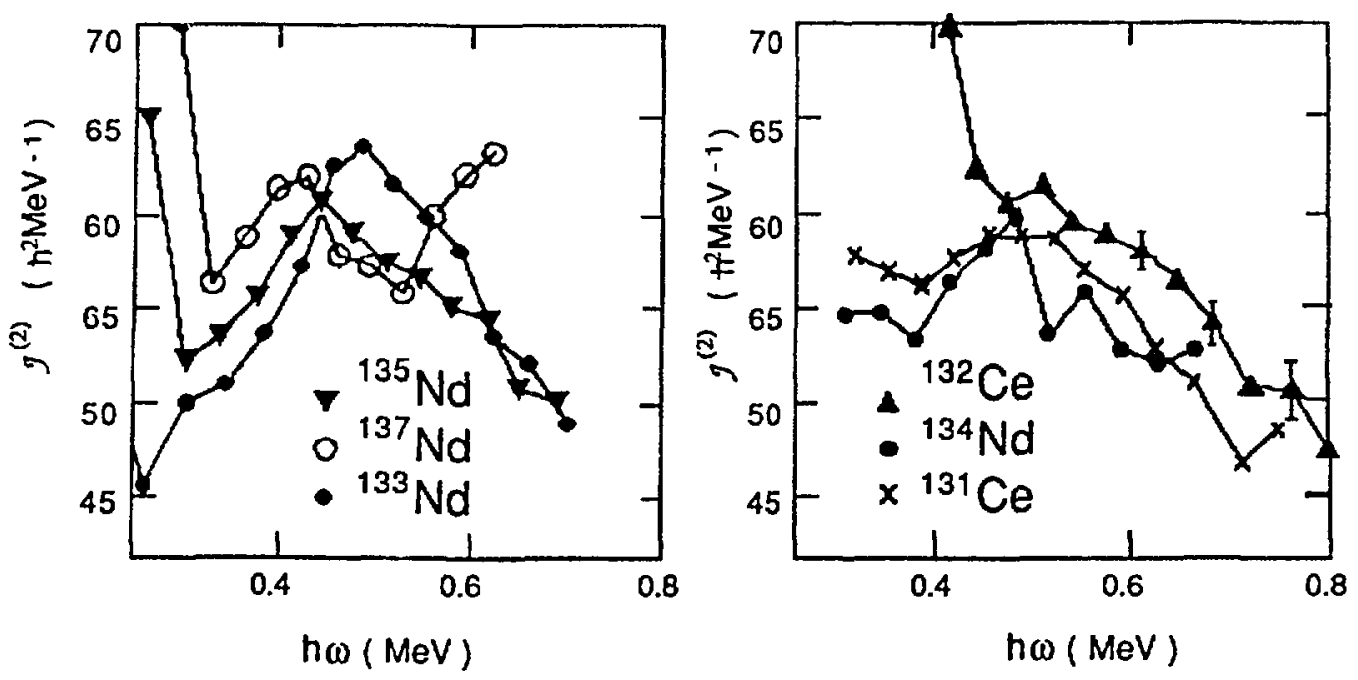

Fig.11 Dynamical moments of inertia for yrast superdeformed bands in the $A \sim 130$ region (This is the same figure (Fig. 8) of Prof. Twin's contribution in these proceedings)

I should emphasize that $A \sim 130$ now appears to be a particularly interesting mass region. For this mass region, fission is much less important and $n_{c}$ is small (see Table 4). Such physical conditions allow a much wider window of $\omega$ to exist. Some measurements 28 and theoretical calculation 34 had been carried out in this mass region. Data are shown in Fig. 11. The results are particularly exciting (especially since it seems to support our predictions). The $g^{(2)}$ pattern for $A \sim 130$ appears very much like Fig. 9 (d) and (e). For the Nd isotopes, there is a sharp rise of $g^{(2)}$ in the low frequency regions. This signals a sharp band crossing at such a frequency, precisely what the DAP band would predict. The situation is less dramatic for $\mathrm{Ce}$, although they also suggest some effects of a band crossing there. Correspondingly, according to Table 4 , $\bar{n}_{c}$ for $\mathrm{Nd}$ is 4 , and for $\mathrm{Ce}$ it is zero, but one must awaked of a one pair uncertainty.

Another relevant and interesting question is why most of the SD nualei in the $A \sim 190$ region have a similar $\mathcal{X}^{2}$ ) behavior (in fact, except for the two excited bands of ${ }^{193} \mathrm{Hg}$, all 
the data fall virtually on the same curve), while for $A \sim 150$ the variations are more dramatic. The SFDSM can also provide an answer. The key point is the different nature of the CAP alignment and the high-j neutron (proton) alignment in the intruder levels. The CAP alignment is a collective pair breaking in the supershell, not a pair breaking in a specific single-particle orbital. It therefore depends cnly on the global structure of the supershell and not the individual orbitals. This should be contrasted with the high- $j$ neutron (proton) alignment which is essentially single- $j$ in nature, and thus is quite sensitive to the configuration of the aligned particles. For the SD nuclei in a given region the configuration for the particles in the intruder levels may differ from nucleus to nucleus, but the supershell configuration remains rather stable. Thus, the part of the $x^{(2)}$ curve sensitive to high-j alignment will in general change from nucleus to nucleus, while the part of $x^{2}$ ) which is sensitive to the CAP alignment will be stable. For $A \sim 190$ all the available data are in the region dominated by the CAP alignment; this explains why all the $x^{2)}$ curves appear to be similar. For $A \sim 150$, all the available data are in the region sensitive to the high-j alignment; this explains why $\left.x^{2}\right)$ in this region varies dramatically. Furthermore, the physics for $A \sim 150$ is close to that depicted by Fig. $9(f)$, which makes $\mathcal{X}^{(2)}$ even more sensitive to the configuration of the aligned particles. Thus, within the SFDSM framework generic features of the moments of inertia of SD bands emerge naturally in terms of a simple, analytical model.

\section{4 "Twinned" bands?}

Several excited SD bands have now been observed. The most intriguing observation is the identical (or "twinned" !) bands in the A 150 region :

$$
\begin{aligned}
& \left.{ }^{I S I} \mathrm{~Tb} \text { (yrast SD band }\right)={ }^{I S 0^{\circ}} \mathrm{Gd}(\text { excited SD band }) \\
& { }^{I 52} \mathrm{Dy}(\mathrm{yrast} \text { SD band })={ }^{I S I} \mathrm{~Tb}(\text { excited SD band })
\end{aligned}
$$

By "twinned bands", one means that the $\gamma$ transitional energies of the two bands are identical to within $\pm 3 \mathrm{keV}$ (see Fig. 5 of Prof. Twin's contribution in these proceedings). The reason why such observations caused a stir is because one of the "twins" is eveneven and the other is odd-A. Generally speaking, even if their core moments of inertia were identical (a surprise in itself), there is no reason to presume the equivalence of their $\gamma$ transitional energies $E_{\gamma}$ unless the decoupling constant $a$ equals unity,

$$
E_{\gamma}^{\text {even }}(J)=(2 J-1) / 2 y_{\text {even }}, \quad E_{\gamma}^{\text {odd }}(J \pm 1 / 2)=(2 J \pm l \mp a-1) / 2 g_{\text {odd }}
$$

Unfortunately, for the standard Nilsson asymptotic quantum numbers for the relevant orbital, $a$ is not unity but vanishes! Recently, Nazarewicz et al. have proposed that instead of the usual Nilsson scheme a pseudo- $\mathrm{SU}_{3}$ scheme is consistent with $a=1$, thereby obtaining the desired results. 30 This presumes that the odd particle (hole) will 
not modify the core moment of inertia. Of course, the assumption $g_{\text {even }}=3_{\text {odd }}$ deserves an explanation, especially because the average $A^{5 / 3}$ dependence of the nuclear moment of inertia expected from the liquid drop model leads to a difference an order of magnitude larger than what is experimentally observed.

Within the SFDSM framework, these identical bands can be understood naturally. According to Table 4, the SD configurations for these SD bands can be taken as follows:

Table 5. The supershell configurations for $N=86$ isotones

\begin{tabular}{ccc}
\hline Nucleus $^{*}$ & Yrast band & Excited band \\
\hline${ }_{64}^{150} G d_{86}$ & $\pi(3)^{0}\left[(4)^{10}(5)^{12}\right]\left(\mathrm{i}_{13 / 2}\right)^{2}$ & $\pi(3)^{\overline{1}}\left[(4)^{10}(5)^{12}\right]\left(\mathrm{i}_{13 / 2}\right)^{3}$ \\
${ }_{65}^{15} \mathrm{~T} b_{86}$ & $\pi(3)^{0}\left[(4)^{10}(5)^{12}\right]\left(\mathrm{i}_{13 / 2}\right)^{3}$ & $\pi(3)^{\overline{1}}\left[(4)^{10}(5)^{12}\right]\left(\mathrm{i}_{13 / 2}\right)^{4}$ \\
${ }_{66}^{152} D y_{86}$ & $\pi(3)^{0}\left[(4)^{10}(5)^{12}\right]\left(\mathrm{i}_{13 / 2}\right)^{4}$ & $\pi(3)^{1}\left[(4)^{10}(5)^{12}\right]\left(\mathrm{i}_{13 / 2}\right)^{5}$ \\
\hline
\end{tabular}

- The neutron configuration is $v(4)^{14}\left[(5)^{14}(6)^{16}\right]\left(j_{15 / 2}\right)^{2}$

From Table 5, one sees that all these nuclei have identical supershell structure, but generally different alignment configurations. The data in this region, as I have pointed out in section 4.3, is more sensitive to the high-j alignment; therefore, even for the same nucleus its excited bands may not be the same as its yrast band. However, for special cases, like ${ }^{152} \mathrm{Dy}$ (yrast SD band) and ${ }^{151} \mathrm{~Tb}$ (excited SD band), or ${ }^{151} \mathrm{~Tb}$ (yrast SD band) and ${ }^{150} \mathrm{Gd}$ (excited SD band), the configurations are the same except that the excited band of ${ }^{151} \mathrm{~Tb}\left({ }^{150} \mathrm{Gd}\right)$ has a proton hole in the hole-shell $\left(\bar{n}_{c}=I\right)$. Thus, according to the SFDSM different hole-shell configuration will affect the region below the decaying out point. The observed range of frequencies of the $f^{(1)}$ and $g^{(2)}$ curves are entirely determined by the supershell structure and the high-j configuration. Hence, it is not surprising that the moments of inertia are nearly identical. In fact, for the SFDSM in the symmetry limit, they mus: be so. Also, the symmetry of the hole-shell in $A \sim 150$ is pseudo-SU3 (see Table 3); thus $a=1$, and one obtains identical bands. In fact, 1 would like to suggest that these identical bands are not really identical. If one can measure the portion of the curve below the decaying point, the difference will show up!

For $A \sim 190$, the decaying out point occurs low in frequency and the high frequency portion is cut off by the fission; thus, the data fall in a region which is not sensitive to the high-j alignment, and even though the high-j configuration is different the moments of inertia for all SD bands should have similar behavior. This situation is indeed observed, as we have discussed. 
Although in our model the pseudo-S $U_{3}$ symmetry does appear for $A \sim 150$, it is special for $N=3$. This is different from the general pseudo- $S U_{3}$ scheme 4,5 where all the normal parity levels in each physical shell are classified by pseudo-spin $1 / 2$. This is particularly interesting since the SFDSM now also appears to be successful in explaining the identical bands (plus the other three important physical features). It is worthwhile to see whether the eritire pseudo- $\mathrm{SU}_{3}$ scheme is required to describe the $\mathrm{SD}$ phenomena.

\section{SUMMARY}

The power of dynamical symmetry is its simplicity. Indeed, if the symmetry is suitable for the physics, then generic features should emerge naturally. The main focus of this talk is to provide a microscopic understanding of deformation. I have shown that the normal deformations can be described as one major shell physics, and are closely related to the $\mathrm{SU}_{3}$ fermion dynamical symmetry. In particularly, the dynamical Pauli effect, which forbids the occurrence of $S U_{3}$ symmetric irreps when $n_{1}>2 \Omega_{1} / 3$, plays a crucial role.

Likewise, superdeformation appears to be closely related to the $\mathrm{SU}_{3}$ dynamical symmetry of a supershell, which consists of two neutron shells and two proton shells. Thus the FDSM is extended to the SFDSM. Again, the dynamical Pauli effect plays an important role. It is remarkable that with this as the guiding principle a map of SD nuclei is produced, and it agrees with all the experimentally verified SD regions. It also predicts some new regions. The especially interesting ones are the oblate SD regions.

I have listed four experimental features of SD (see Section 3 ) as a challenge to this model. In order for the model to survive, these features must emerge in a unified manner. The preliminary indication is that they do.

This model (SFDSM) reveals two new ingredients of the superdeformation: a strong interaction between high-j orbital and supershells and a weakly interacting DPA band as the SD ground band. I showed that a simple 3-band simulation with such new ingredients included essentially captured all the experimental features of the SD physics in the mass regions of $A \sim 130,150$ and 190. It would be especially interesting to see whether one could detect the states below the decaying out point. Perhaps this is a question best posed to new experimental detecting systems, like the GAMMASPHERE.

I would like to end my talk by posing an intriguing question: if the SFDSM $\mathrm{SU}_{3}$ limit corresponds to $S D$, then to what does the $\mathrm{SU}_{2}$ limit correspond? Is it possible that the physics inherent in this limit is related to the giant quadrupole resonances one observes in nature? This is currently an open question, and it will certainly be interesting and worthwhile to pursue its answer! 
My colleagues and $I$ are very grateful for the useful discussions we had with $B$. $R$. Mottelson, P. J. Twin, F. S. Stephens, R. M. Diamond, J. Rasmusson, L. L. Riedinger, J. A. Cizewski, T. L. Khoo, U. Garg, J. D Garrett, W. Nazarewicz, P. Ring, S. Pittel, Y. S. Chen, and J. P. Draayer. This research is supported by the U. S. National Science Foundation, the U.S. Department of Energy under contract no. DE-ASO5-76ER04936 and the Chinese Science Foundation.

\section{REFERENCES}

1) C. -L WU, D. H. Feng, X.-G. Chen, J. Q. Chen and M. W. Guidry, Phys. Lett. B168 (1986) 313; Phys. Rev. C36 (1987) 1157 ; C.-L. Wu and D. H. Feng, Workshop on Microscopic Models in Nuclear Structure Physics, Edited by M. W. Guidry, J. H. Hamilton, D. H. Feng, and J. B. McGrory, World Scientific (1988) 21,74.

2) Dynamical Groups and Spectrum Generating Algebras, edited by A. Barut, A. Bohm and Yuval Ne'eman (World Scientific, 1988).

3) J.P. Elliott, Proc. Roy. Soc. A245 (1958)128; ibid. A245 (1958) 562.

4) A. Arima, M. Harvey and K. Shimizu Phys. Lett. B30 (1969) 517.

5) K.T. Hecht, see lecture delivered at the VIIIth Symposium on Nuclear Physics, Notas Fisica, 8 (1985) 165 and refs. therein.

6) G. Rosensteel and D. J. Rowe, Phys. Rev. Lett. 38 (1977) 10; Ann. Phys 126 (1980) 343.

7) J. P. Draayer, Proceedings of Conference on Nuclear Structure in Nineties, Oak Ridge, April (1990).

8) A. Arima and F. lachello, Ann. Rev. of Nucl. Part. Sci. 31(1981) 75.

9) J. N. Ginocchio, Phys. Rev. B79, 173(1978); Ann. of Phj:s. 126 (1980) 234.

10) M. Kirson, in Proceedings of the International Conference on the Comtemporory Topic in Nuclear Structure Physics, Cocoyoc, Mexico, 1988 (World Scientific, 1988, Singapore).

11) J.-Q. Chen, D. H. Feng and C. -L. Wu, Phys. Rev. C34 (1986) 2269; Z.-M. Lü, X.W. Pan, J. -Q Chen, X.-G. Chen and D. H. Feng, Phys. Rev. C37 (1988) 2789.

12) H. Wu, D. H. Feng, C.-L. Wu, and M. W. Guidry, Phys. Lett. B198 (1987) 119 ; H. Wu, D. H. Feng, C.-L. Wu, Z.-P. Li and M. W. Guidry, Phys. Lett. B193 (1987) 163; H. Wu, C.-L. Wu, D. H. Feng and M. W. Guidry, Phys. Rev. C 37 (1988) 1739.

13) W.-M Zhang, D. H. Feng and W. Gilmore, Rev Mod. Phys. (in press).

14) W.-M. Zhang, C. -L. Wu, D. H. Feng, J. N. Ginocchio and M. W. Guidry, Phys. Rev. C38 (1988) 1475; Nucl. Phys. A505 (1988) 7.

15) R. F. Casten, C.-L. Wu, D. H. Feng, J. N. Ginocchio and X.-L. Han, Phys. Rev. Lett. 56 (1986) 2578. 
16) D. H. Feng, C. L. Wu, M. W. Guidry and Z. P. Li, Phys. Lett, B 205 (1988) 157.

17) C. L. Wu, X. L. Han, Z. P. Li, M. W. Guidry and D. H. Feng, Phys. Lett. B194(1987) 447.

18) M. W. Guidry, C.-L. Wu, Z.-P. Li, D. H. Feng and J. N. Ginocchio, Phys. Lett. B187 (1986) 210 ; C.-L. Wu, Proceedings of Conference on Nuclear Structure in Nineties, Oak Ridge, April (1990).

19) H. Wu, C.-L. Wu, D. H. Feng and M. W. Guidry, Phys. Rev. C37 (1988) 1739 ; L. -A. Wu et al. (to be published).

20) Z.-P Li et al. (to be published).

21) C.-L Wu, M. W. Guidry, D. H. Feng and J.-Q. Chen, Fizika 22(1990)1.

22) C.-L. Wu et al. (to be published).

23) J.-Q. Chen, X.-G. Chen, D. H. Feng, C. L. Wu, J. N. Ginocchio and M. W. Guidry, Phys. Rev. C40 (1989) 2844 ; J.-L. Ping et al. (to be published).

24) M. W. Guidry, C.-L. Wu, D. H. Feng, J. N. Ginocchio, X.-G. Chen and J.-Q. Chen Phys. Lett. B176(1986) 1.

25) W.-M. Zhang, D. H. Feng, C.-L. Wu, and M. W. Guidry, J. Phys. G: Nucl. Part. Phys. 15 (1989) L115; L. -A. Wu et al. (to be published).

26) H. Wu and M. Vallieres, Phys. Rev C39 (1989)1066.

27) Z.-P. Li et al. (to be published)

28) P. J. Nolan and P. J. Twin, Ann. Rev. Nucl. Part. Sci. 38 (1988) 533.

29) W. Nazarewicz, R. Wyss and A. Johnson, Nucl. Phys. A503 (1989) 285.

30) W. Nazarewicz, P. J. Twin, P. Fallon and J. D. Garrett, Univ. of Liverpool preprint, 1990.

31) R. R. Chasman, Phys. Lett. B219 (1989) 227.

32) M. A. Riley at al. Nucl. Phys. A512 (1990)178.

33) P. Fallon et al.,Phys. Lett. B218 (1989)137.

34) R. Wyss, J. Nyberg, A. Johnson, Phys. Lett. B215 (1988) 211; K. Tanabe, see contribution to theses proceedings.

\section{DISCLAIMER}

This report was prepared as an account of work sponsored by an agency of the United States Government. Neither the United States Government nor any agency thereol, nor any of their employees, makes any warranty, express or implied, or assumes any legal liability or responsibility for the accuracy, completeness, or usefulness o: any information, apparatus, product, or process disclosed, or represents that its use would not infringe privately owned rights. Reference herein to any specific commercial product, process, or service by trade name, trademark, manufacturer, or otherwise does not necessarily constitute or imply its endorsement, recommendation, or favoring by the United States Government or any agency thereof. The views and opinions of authors expressed herein do not necessarily state or reflect those of the 